Flanagan, K. J.; Bernhard, S. S. R.; Plunkett, S.; Senge, M. O. (2019):

Not Your Usual Bioisostere: Solid State Study of 3D Interactions in Cubanes.

Chemistry_A European Journal 25, 6941-6954. doi: 10.1002/chem.201901523

WILEY-VCH

\title{
Not Your Usual Bioisostere: Solid State Study of 3D Interactions in Cubanes
}

\author{
Keith J. Flanagan, ${ }^{[a]}$ Stefan S. R. Bernhard, ${ }^{[a]}$ Shane Plunkett, ${ }^{[a]}$ and Mathias O. Senge ${ }^{*[a]}$
}

\begin{abstract}
Previous studies by Desiraju and co-workers have implicated the acidic hydrogen atoms of cubane as a support network for hydrogen-bonding groups. Herein we report a detailed structural analysis of all currently available 1,4-disubstituted cubane structures with an emphasis on how the cubane scaffold interacts in its solid-state environment. In this regard, the interactions between the cubane hydrogen atoms and acids, ester, halogens, ethynyl, nitrogenous groups, and other cubane scaffolds were cataloged. The goal of this study was to investigate the potential of cubane as a substitute for phenyl. This could be achieved by analyzing all contacts that are directed by the cubane hydrogen atoms in the Xray crystal structures. As a result, we have established several new cubane interaction profiles, such as the catemer formation seen in esters, the preferences of halogen-hydrogen contacts over direct halogen-bonding, and the stabilizing effects caused by the cubane hydrogens interacting with ethynyl groups. These interaction profiles can then be used as a guide for designing cubane bioisosteres of known materials and drugs containing phenyl moieties.
\end{abstract}

\section{Introduction}

Cubane, due to its inherent 3D geometry offers intriguing possibilities for interaction studies. In 1992, Eaton postulated that cubane, due to its size and shape, would make an ideal bioisostere for a phenyl ring. ${ }^{[1]}$ In this regard, there has been plenty of research into the use of cubane-containing molecules as pharmaceutical and agrochemical compounds. ${ }^{[2]}$ This was further compounded by Chalmers et al. in their validation of Eaton's postulate which highlighted the fact that cubane could act as a bioisostere for phenyl. ${ }^{[3]}$ Another application for cubane is as a rigid linker in materials chemistry. ${ }^{[4]}$ By considering both drug design and materials science, how a molecule interacts with its environment is of prime importance to shape, functionality, and potential contacts. Knowing how a drug which has been modified interacts with its environment is one of the keys to drug design and as such an important path to crystal

[a] K. J. Flanagan, Dr. S. S. R. Bernhard, Dr. S. Plunkett, Prof. Dr. M. O. Senge,

School of Chemistry, SFI Tetrapyrrole Laboratory,

Trinity College Dublin, The University of Dublin,

Trinity Biomedical Sciences Institute,

152-160 Pearse Street, Dublin 2, Ireland.

E-mail: sengem@tcd.ie

Supporting information for this article is given via a link at the end of the document.

CCDC 1887763-1887774 contain the supplementary

crystallographic data for this paper. These data can be obtained free of charge from The Cambridge Crystallographic Data Centre via www.ccdc.cam.ac.uk/data request/cif.

engineering. With this in mind, knowing how cubane interacts with its environment is as prudent as much as knowing if cubane is structurally similar to phenyl when considering it as a bioisostere. Therefore, an interactive study into the structure of cubane and its functionalized derivatives is warranted and with over 100 structure datasets of 1,4-disubstituted cubane structures available in the CCDC database the time for such a study is ideal.

a) syn-anti catemer

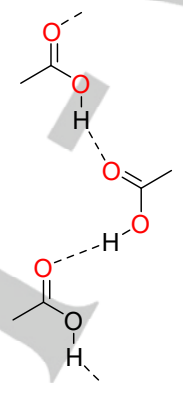

c) Cyclic pattern for $\mathrm{R}=\mathrm{COOCH}_{3}$
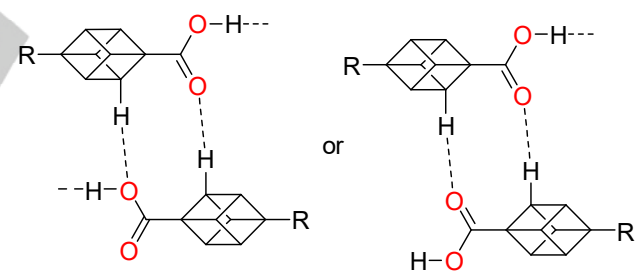

Figure 1. Different hydrogen-bonding interactions for 1,4-substituted cubane carboxylic acids. ${ }^{[5]}$

Previous studies by Desiraju and co-workers on crystal engineering of cubane scaffolds focused on the series of 4-(R)substituted-1-cubanecarboxylic acids. ${ }^{[5-6]}$ Within this series, they demonstrated the presence of rare syn-anti catemers in the formation of the hydrogen-bonds (Figure 1). This was theorized to be a direct result of a secondary binding motif between the cubane hydrogen and carbonyl groups leading to a more favored syn-anti catemer formation. Additionally, when halogens were incorporated into the 4-position of the cubane scaffold they were seen to occupy a centrosymmetric void present in these structures further stabilizing the network. When an ester $\left(\mathrm{CO}_{2} \mathrm{Me}\right)$ is placed in the 4-position of the cubane scaffold a noticeable shift is observed towards the formation of cyclic patterns with the ester partaking in hydrogen-bonding with the hydrogen of the cubane scaffold. Moreover, in cases where the 4-position is small $(\mathrm{H})$ or large $(\mathrm{Ph})$, or when an equally competitive hydrogen-bonding partner is included $\left(\mathrm{CONH}_{2}\right)$, the 
catemer was no longer formed and a more classical dimer observed.

A further study investigated how the $\mathrm{C}-\mathrm{H}$ bond of cubane is activated toward hydrogen-bond formation and its ability to support a framework with stabilizing $\mathrm{C}-\mathrm{H} \cdots \mathrm{O}$ bonds. ${ }^{[6 b]}$ Initially, the authors chose to work with primary cubane carboxamides to observe the formation of $\mathrm{N}-\mathrm{H} \cdots \mathrm{O}$ hydrogen-bonds. ${ }^{[7]}$ Similar to what is observed in carboxylic acid dimers, the common motif observed for primary carboxamides is the centrosymmetric dimer with syn-oriented $\mathrm{N}-\mathrm{H}$ groups. Additionally, anti-oriented $\mathrm{N}-\mathrm{H}$ groups can be seen to form either a linear pattern with or without a glide plane (shallow-glide motif). The motif without the glided plane characteristically is related by $5.1 \AA$ translation to the next succeeding molecule. When a linear pattern without a glide plan occurs, the dimer motif of the commonly occurring translational ribbon synthon is formed. Notably, the primary cubane carboxamides have been shown not to utilize the ribbon motif, because the cubane skeleton is considered too bulky for this translation motif resulting in the formation of the less sterically demanding shallow glide conformation. As before, it appears that this conformation is further reinforced by the formation of a hydrogen-bond between oxygen atoms and the cubane hydrogen (Figure 2). This ability is attributed to the large $\mathrm{C}-\mathrm{C}-\mathrm{H}$ pyramidality angle of the interacting hydrogen atoms present due to sterically restricted nature of the tertiary carbon atoms ${ }^{\left[{ }^{[8]}\right.}$ Other structural reports on cubane scaffolds exist in the literature, however, none go into quite as much detail of potential contacts bar those outlined above. With this in mind, we performed broad scale crystallographic studies, reporting 12 new cubane structures and, together with data from the CCDC investigated the range of interactions available to cubane scaffolds and their potential implications in the field of crystal engineering.

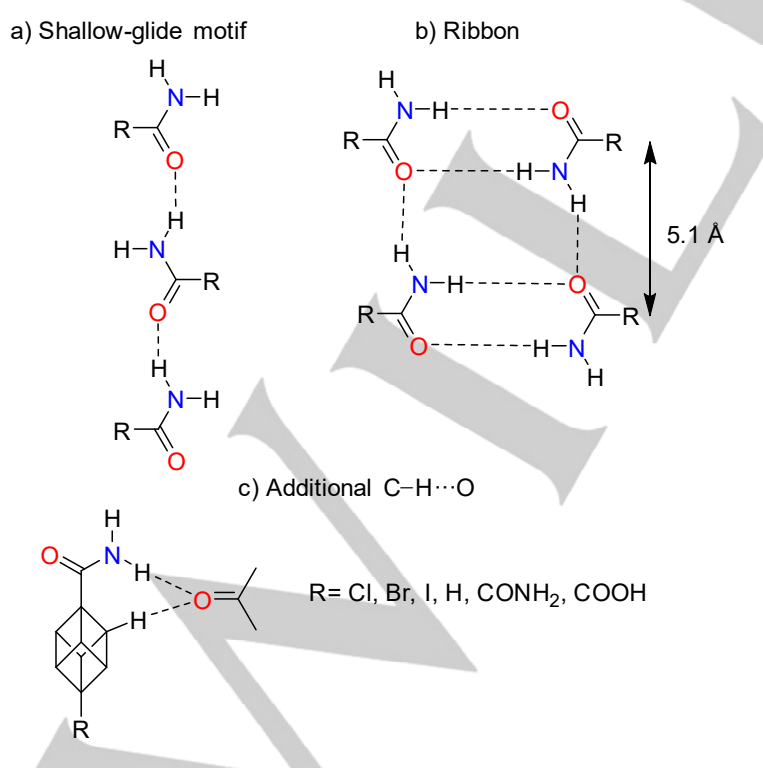

Figure 2. Amide hydrogen-bonding motifs. ${ }^{[7]}$

\section{Results and Discussion}

\section{Benzene vs. cubane}

As mentioned previously, cubane has been used to an extent as a bioisostere. This topic was covered by Chalmers et al. among others where cubane containing drugs showed equal or increased bioactivity for in vivo or in vitro tests. ${ }^{[2-3,9]}$ In these studies, the authors have focused on solubility, stability, tractability and the availability of suitable precursors as the main concerns for using cubane as a bioisostere for a benzene ring. ${ }^{[3]}$ However, when considering the shape of cubane and benzene, there is a world of differences between a flat molecule, i.e. benzene and the 3D architecture of cubane. As discussed by Luh et al. the kinetic acidity of cubane is similar to that of benzene. ${ }^{[10]}$ Therefore, both compounds have an equal potential to form $\mathrm{C}-\mathrm{H} \cdots \mathrm{A}$ contacts (where $\mathrm{A}$ is any hydrogen acceptor). However, cubane has two more hydrogen atoms that can participate in $\mathrm{C}-\mathrm{H} \cdots \mathrm{A}$ contacts and the orientation of the hydrogen atoms are very different to that of benzene.

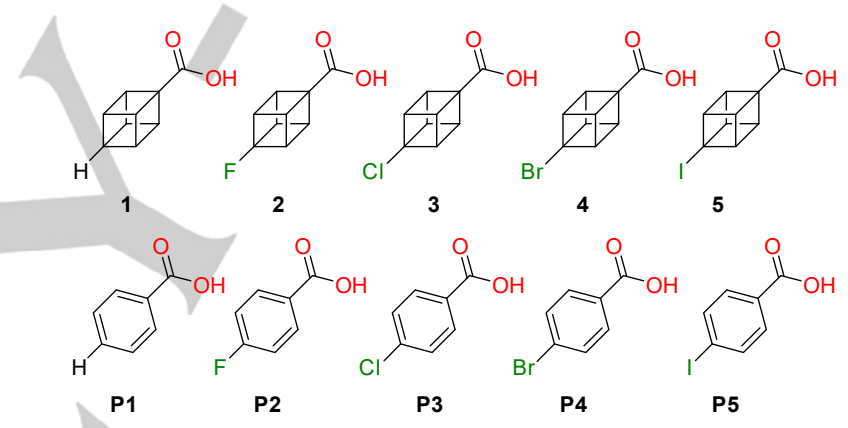

Figure 3. Literature structures of 1-carboxylic acid-4-(R)-cubane and 4-(R)benzoic acid (where $\mathrm{R}$ is $\mathrm{H}, \mathrm{F}, \mathrm{Cl}, \mathrm{Br}$, or I). ${ }^{[6,11]}$

As a small case study, we examined the interaction profiles of 1 carboxylic acid-4-(R)-cubane and 4-(R)-benzoic acid (where $R$ is $\mathrm{H}, \mathrm{F}, \mathrm{Cl}, \mathrm{Br}$, or I) to assess their differences (Figure 3 and 4$)$. In Figure $4(B, D, F, H$, and $J)$ there is a clear trend in the 4-(R)benzoic acid compounds where the carboxylic acid moieties all form head-to-head dimers. The benzene hydrogen atoms are also seen to have favorable interactions with the carboxylic acid moiety forming a linear $\mathrm{C}-\mathrm{H} \cdots \mathrm{O}$ network. This motif is preserved in all examples and only varies by the interactions of the substituent at the para-position of the benzene moiety with either halogen $\cdots$ halogen or halogen $\cdots$ hydrogen interactions observed. This is not the case for the cubane derivatives. In Figure 4 (A, C, $E, G$, and I), there is no standard pattern observed and each $C$ $\mathrm{H} \cdots \mathrm{O}$ network in compounds $1-6$ is unique in the crystal packing. This shows that as the cubane scaffold has both more hydrogen atoms and $3 \mathrm{D}$ orientation incorporated the potential for unique packing patterns is increased dramatically. In the case of the 4(R)-benzoic acid there is a clear case of predictability in the interaction profile. Whereas, cubane, due to the lack its inherent properties, makes predicting the interactions profile much harder. This leads to the current problem of using cubane as a 
bioisostere for a benzene or a phenyl ring. While both molecules may be of similar size, they are not of similar interaction profiles. Basing such studies on this concept relies more on trial and error than it does on design, which highlights the importance of understanding the potential interactions between the cubane hydrogen atoms and appropriate acceptor groups.
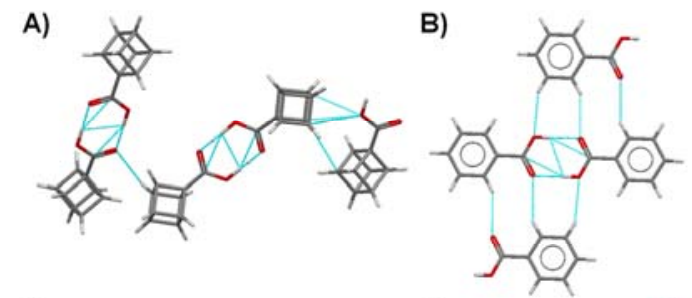

C)

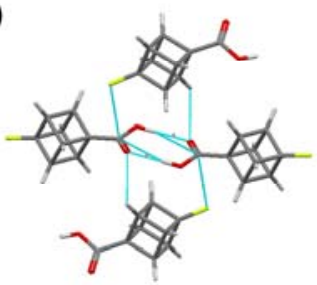

E)

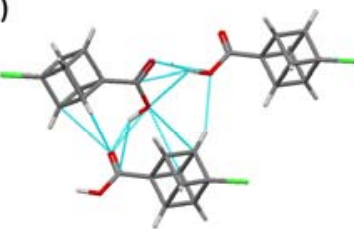

G)

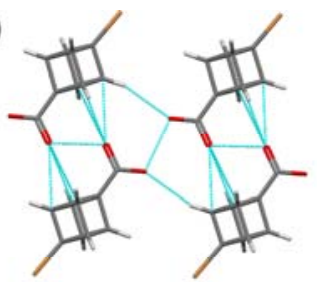

I)

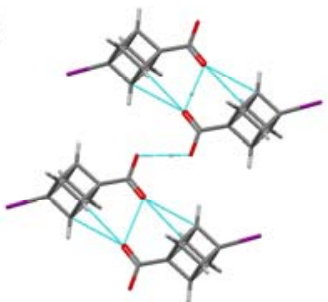

D)

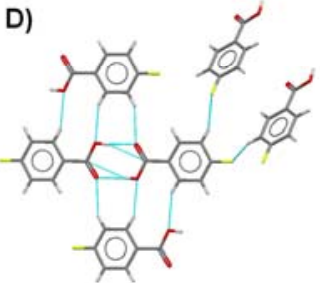

F)

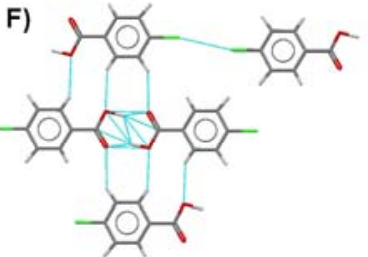

H)

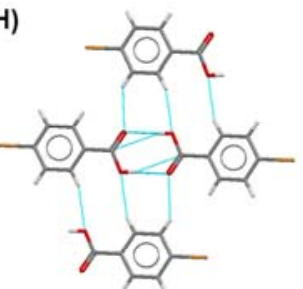

J)

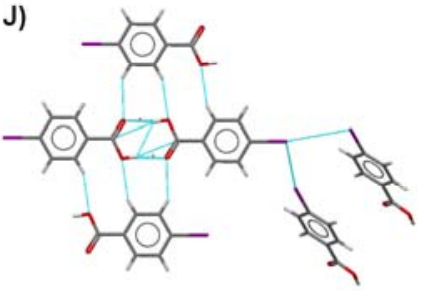

Figure 4. View of the molecular arrangement in the crystal of compounds 1 (A), P1 (B), 2 (C), P2 (D), 3 (E), P3 (F), 4 (G), P4 (H), 5 (I), and P5 (J) showing the hydrogen-bonding between the carboxylic acid and the cubane/benzene hydrogen atoms. Interactions are indicated by dashed blue lines.

\section{Carboxylic acids}

The first series to be discussed is the 1-carboxylic acid-4-(R)cubane structures that were previously reported by Desiraju and co-workers. ${ }^{[6 a]}$ As outlined in the introduction carboxylic acid derivatives of cubane tend to favor the formation of syn-anti catemers or at least this motif is more prevalent in cubane crystal structures than their benzene counterparts. (Figure 5, bond lengths and angles are given in Table S1). ${ }^{[6,12]}$ In these compounds (also for the carboxamides and esters sections below) hydrogen-bonding is one of the main driving forces seen in the packing. Hydrogen-bonding is simply defined (by IUPAC standards) as an attractive interaction between a hydrogen atom from a molecule or a molecular fragment that is more electronegative and an atom or group of atoms in the same or different molecule where there is evidence of bond formation. ${ }^{[13]}$ The bond distance typically is less than or equal to the sum of the Van der Waals radii of the atoms involved and the angles depend on the atoms involved. These standards are also followed for non-classical hydrogen-bonding. ${ }^{[14]}$
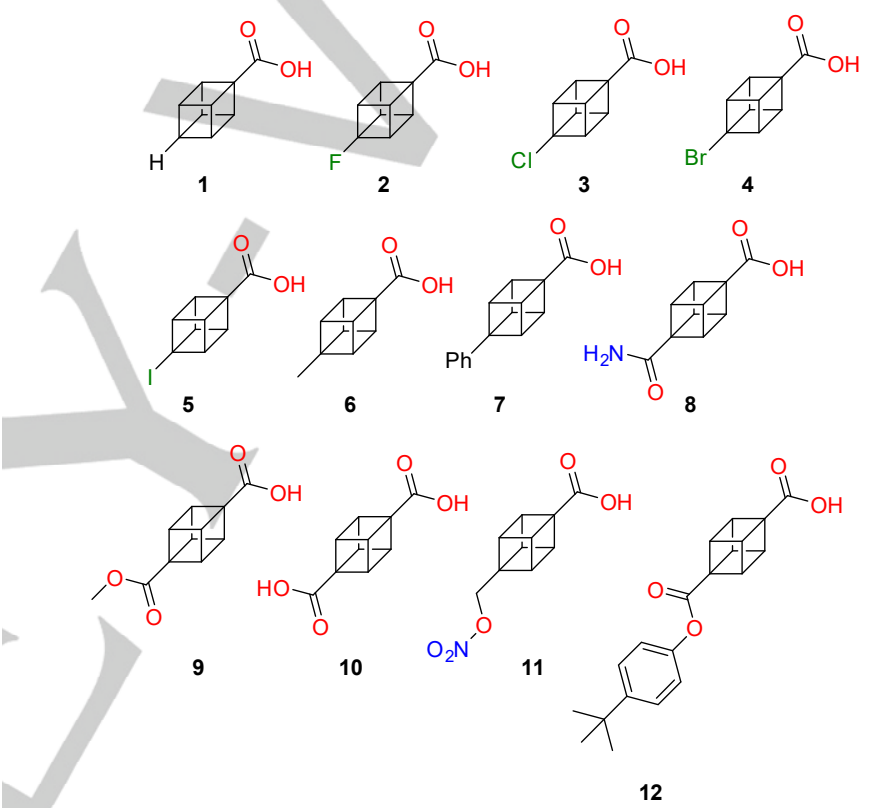

Figure 5. Available literature structures of 1,4-substituted cubane carboxylic acid derivatives. ${ }^{[6,12]}$

From this series, it is interesting to note that 6 out of 11 of the published structures favor this syn-anti catemer formation. Considering the average occurrence for this motif in the literature is quite low this high average within a specific family is quite unique. ${ }^{[6 a]}$ Das and Desiraju have previously stated that the propensity for this motif is stabilized by a supportive $\mathrm{C}-\mathrm{H} \cdots \mathrm{O}$ interaction. ${ }^{[5]}$ It appears that the natural 3D conformation of cubane containing six available $\mathrm{C}-\mathrm{H}$ interactive groups in a spatially defined pattern increases the potential of these contacts. As highlighted by Fleischer et al. the bent bonds of cubane scaffold allow for the acidic hydrogen atoms to be presented in several directions which are unavailable for its benzene counterpart. ${ }^{[15]}$ However, this effect is only seen in certain cubane carboxylic acids (3-6,9 and 10). It can be seen that the 4-substituted position to the cubane scaffold plays an important role with the catemer formation. In cases where the 4residue is small (1 and $\mathbf{2}$ ) or large (7), or when an equally 
competitive hydrogen-bonding partner is included (8), the catemer is no longer formed and a more classical dimer is observed. Additionally, when looking at the structure of compound $\mathbf{1 1}$ there is an interesting development as the $\mathrm{NO}_{2}$ group has now taken over interacting with the cubane scaffold, resulting in the formation of the common dimer system. Further inspection of the crystal structures of 1-11 shows that when the syn-anti catemer is formed the 4-substituted position interacts less with the acidic hydrogens of the cubane scaffold than when the syn-anti catemer is not formed. This suggests a possible competition between these interactions.

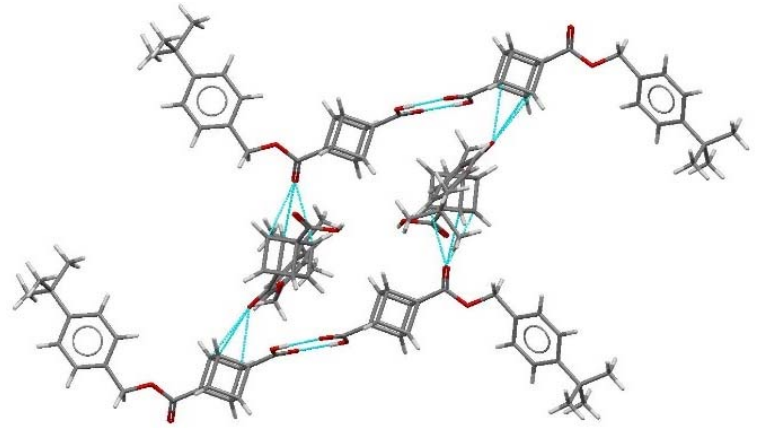

Figure 6. View of the molecular arrangement in the crystal of compound 12 showing the hydrogen-bonding between the carboxylic acid and the cubane ester interactions resulting in rotated layers. Interactions are indicated by dashed blue lines.

One new structure is herein added to the list of 1,4-substituted cubane carboxylic acid structures. The structure of 12 exhibits head-to-head dimer formation as expected due to the relatively large size of the 4-position substitution (Figure 6). There is no evidence of catemer formation within the structure and the presence of a $\mathrm{C}-\mathrm{H} \cdots \mathrm{O}$ contact between the ester and the cubane scaffold results in an almost orthogonal rotation between the crystal layers in the unit cell. This is due to the acid dimer and the ester cubane interactions that the structure forms a mesh-like hydrogen-bonded network in two directions.

\section{Carboxamides}

When looking at alternate hydrogen-bonding groups such as the 1,4-substituted cubane carboxamide derivatives structures shown in Figure 7 (for bond lengths and angles see Table S2) an alternate hydrogen-bonding network is observed. ${ }^{[6 a, 7]}$ Due to the nature of these groups $(8,13-15)$ and the strong hydrogenbonding properties of carboxamides, dimer formation is much more prevalent and thus, there is no formation of the syn/anti catemers resulting in a greater number of interactions between the 4-position and the cubane scaffold. As shown by Kuduva et al. this class of structure adopts a dimer formation combined with a shallow glide motif, which is unusual for carboxamides. ${ }^{[7]}$ However, it was highlighted that due to the steric bulk of cubane, the average sectional distance of $5.4 \AA$ is too large to fit into a $5.1 \AA$ translational motif. What is equally interesting as a counterpoint to 1-carboxylic acid-4-(R)-cubanes, the 4- substituted position always aids in the formation of this shallow glide motif through halo-cubane/hydrogen-cubane interactions. Interestingly, the structure of $\mathbf{8}$ forms the only head-to-tail dimer present in this set which results in an a-glide motif.

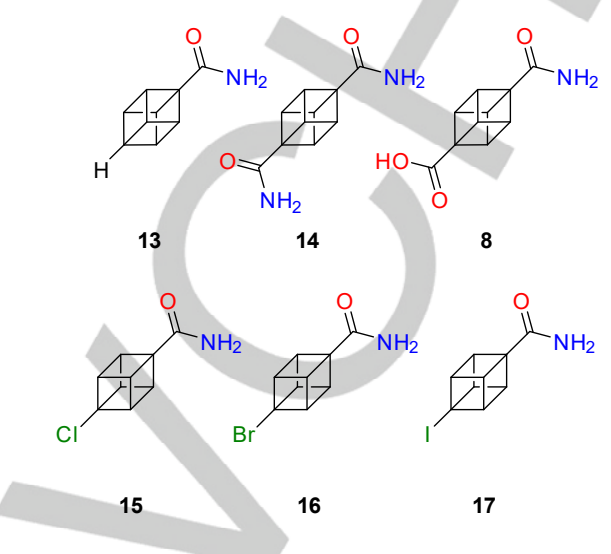

Figure 7. Available structures of 1,4-substituted cubane carboxamide derivatives.

\section{Esters with cubanes}

To this point, we have discussed interactions that have been previously described by Desiraju and co-workers; however, a section that has not been covered to date is the connections between cubane and esters. To date, there have been 27 published structures of cubane bearing esters in the 1-position in the CCDC database (Figures 8, 10 and 12 and data in Table S3). ${ }^{[4 a, 6 a, 16]}$ For simplicity, in each structure the oxygen atoms that are involved in each contact are numbered in Figures 6, 8, and 10 to allow clear indication of the atoms being discussed. While other functionalities are present in these structures, their interactions and effects shall be discussed only briefly here, but in more detail in their relevant sections. Esters themselves provide a very interesting case. Generally, they cannot perform as a hydrogen-bond donor due to a lack of suitable hydrogen to donate; however, they can and do participate frequently as hydrogen-bond acceptors. Previously we have published on this with very simple molecules, such as 4(methoxycarbonyl)phenylboronic acid, in which the methyl ester is clearly seen to participate as a hydrogen-bond acceptor to create an alternate zig-zag stacking pattern compared to its carboxylic acid derivative. ${ }^{[17]}$ For cubane, this aspect offers much more potential due to the acidic hydrogens of the cubane scaffold to act as hydrogen-bond donors in a non-classical fashion. This results in a large number of interactions between cubane hydrogen atoms and ester groups in a hydrogenbonding fashion that can be identified (Table S3). The first section is the methyl esters as outlined in Figure 8 , which consists of mainly simple methyl ester in the 1-position of the cubane scaffold. When looking at 1-(methoxycarbonyl) cubane (18) there is a short contact between $\mathrm{O}^{1}$ and the cubane hydrogen atoms which is compounded by a short contact between $\mathrm{O}^{2}$ and the methyl hydrogen atoms (Figure 9A).[6b] These connections result in a linear network between the 
cubane molecules directed by the ester group. When moving to more functionalized cubane scaffolds such as the halogen series 19-22 we see that this trend is preserved (Figure 9A-E). ${ }^{[16 \mathrm{~h}]}$ The halogens themselves partake in certain directive bonds (see halogen section below) but the main linear network of cubane ester connection is preserved. However, $\mathbf{2 1}$ appears to deviate from this trend slightly favoring a more head-to-tail overlap between cubane and the methyl ester. ${ }^{[16 \mathrm{~h}]}$
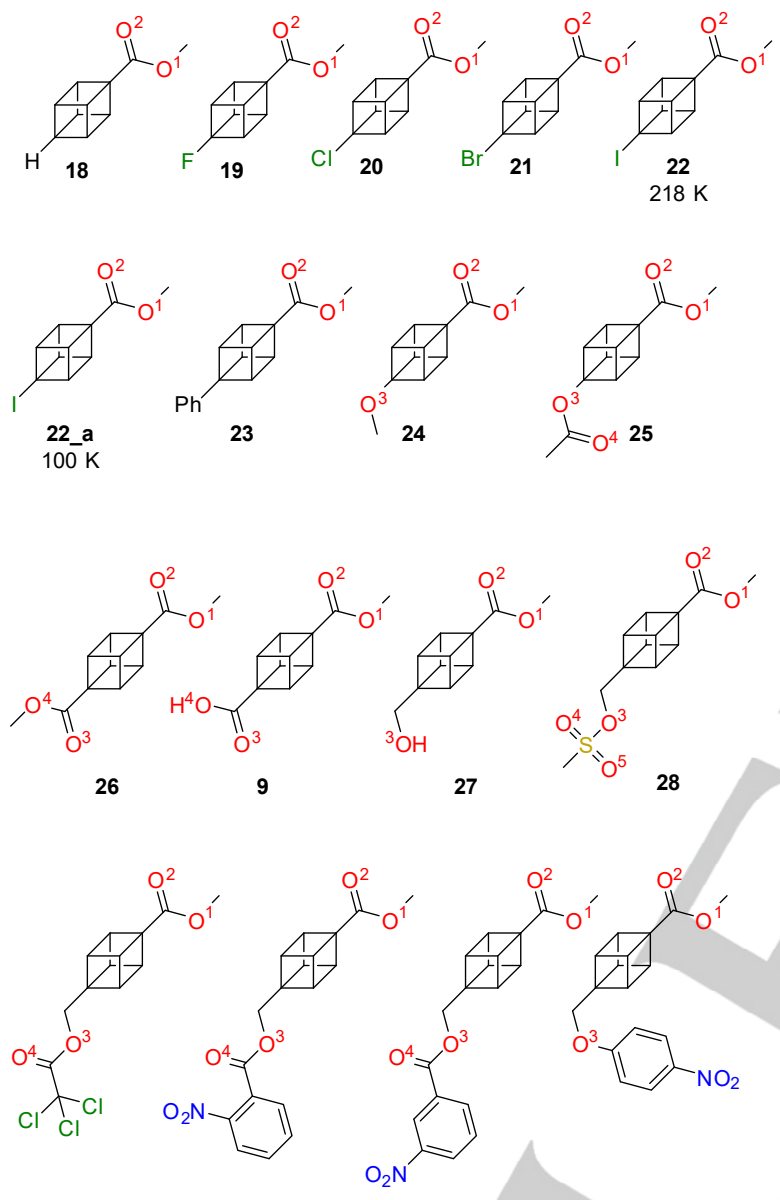

29

30

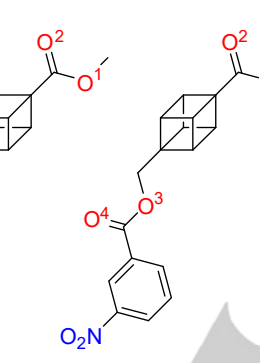

31

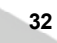

Figure 8. List of methyl ester containing 1,4-disubstituted cubane structures.

The structure of 22_a (which is the same as 22) shows a slight difference to its previously determined counterpart. The literature structure 22 was determined at $218 \mathrm{~K}$ whereas the structure of 22_a was determined at $100 \mathrm{~K}$. This results in a shift in cell axes $(a=24.986, b=6.551, c=5.777 \AA$ for 22 and $a=15.493, b=$ $7.129, c=8.394 \AA$ for 22_a) due to a change in space group from Pnma (22) to $P 2{ }_{1} / C(22$ a). This translates to a shift in short contacts between the structure with 22_a now showing much more overlap between the ester units with $\mathrm{C}-\mathrm{H}_{\text {cubane }} \cdots \mathrm{C}$ interactions between the methyl moiety and the cubane scaffold (Figure S1). The structure of $\mathbf{2 3}$ shows a different alternative in which the ester $\mathrm{O}^{2}$ now interacts with the methyl hydrogen atoms rather than the cubane scaffold, suggesting that competition for hydrogen contacts is possible with alternate groups (Figure S2). ${ }^{[16 f]}$

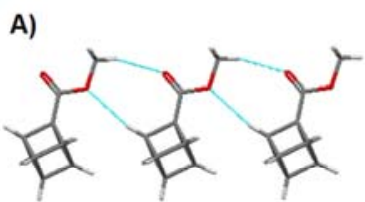

C)

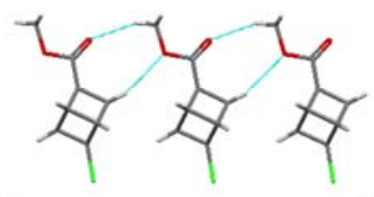

E)

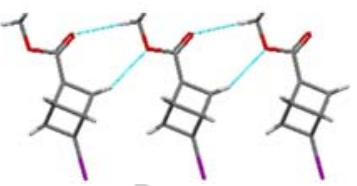

B)

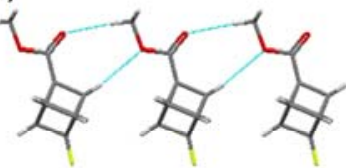

D)

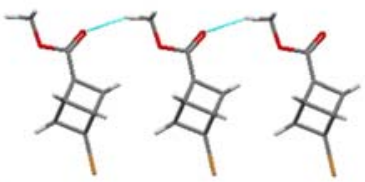

F)

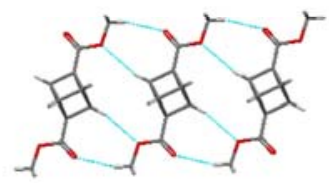

Figure 9. View of the molecular arrangement in the crystal of compound $\mathbf{1 8}$ (A), $19(B), 20(C), 21(D), 22(E)$, and $26(F)$ represented as stick models. Each view shows the preference for ester interactions within the given structure. Interactions are indicated by dashed blue lines.

The structure of 26 (1,4-dimethoxycarbonyl-cubane) preserves the motif seen in $\mathbf{1 8}$ but this time it is twofold on both sides of the cubane scaffold (Figure 9F). ${ }^{[16 c]}$ However, by simply substituting one side of the cubane scaffold with a methyl ether (24) $\mathrm{O}^{2}$ becomes the dominant side at which the cubane hydrogen atoms interact with the ester moiety. ${ }^{[16 a]}$ Additionally, the methyl ester is involved in a reciprocal dimer formation with the methyl ester of its nearest neighbor, changing the packing from a linear network to a twofold network of head-to-head dimers (Figure S3). When substituted with a carboxylic acid (9) on one side of the cubane scaffold we not only see the cubane participate in catemer formation as mentioned above, we see a bifurcated interaction between $\mathrm{O}^{2}$ and the cubane hydrogen (Figure S4). ${ }^{[6 a]}$ Substitution with an acetoxy group (25) shows a similar network to 26; however, instead of the ester group involved in the bond with the cubane hydrogens, the acetoxy group appears to be more dominant with $\mathrm{O}^{3}$ and $\mathrm{O}^{4}$ involved in the formation of a linear network (Figure S5). ${ }^{[16 a]}$ The ester side shows more preference in forming dimers like $\mathbf{2 4}$ and an additional reciprocated bifurcated contact between the hydrogen atoms of the terminal carbonyl group. In the structure of compound 27, the hydroxymethyl groups form a type of catemer partially stabilized by cubane hydrogen atoms interacting with $\mathrm{O}^{3} .{ }^{[161]}$ There is also the appearance of a short hydrogen contact between $\mathrm{O}^{2}$ and cubane hydrogen to form a simple linear network. Both of these interaction motifs together form a rather complex looking packing system (Figure S6). When a methyl methanesulfonate is inserted to the 4-position (28) of the cubane scaffold there is a preference for forming a head-to-tail overlapped network of cubane hydrogen atoms interacting with 
the $\mathrm{O}^{2}$ on one side and $\mathrm{O}^{1}$ on the other which is repeated to form this network. ${ }^{[161]}$ The $4-(($ methylsulfonyl)oxy)methyl moiety has several contacts, one of these being an interaction between $\mathrm{O}^{4}$ and the cubane hydrogen (Figure S7). However, it is more common to see the methyl ester interacting with the cubane than the 4-((methylsulfonyl)oxy)methyl unit. The introduction of a 4((2,2,2-trichloroacetoxy)methyl) group (29) results in cubane hydrogen interacting with $\mathrm{O}^{1}$ forming a linear network in a headto-head fashion and with $\mathrm{O}^{2}$ in a head-to-tail overlapped dimer (Figure S8). ${ }^{[16]]}$ Additionally, the chlorine atoms can interact with the cubane scaffold and other chlorine atoms in a halogenbonding fashion to form head-to-head dimers. The structure of 30 features an ortho-nitrobenzoyloxy group at the 4-position of the cubane scaffold. ${ }^{[161]}$ There are two interactive group types here, namely the ester type (Figure S9_left) and the nitro type (Figure S9_right). The ester type bond is centered on $\mathrm{O}^{4}$ oxygen atom to the cubane hydrogen and is aided by the $\mathrm{O}^{2}$ oxygen interacting with a benzene hydrogen to form an alternating pattern of head-to-head dimers. The nitro group acts in a similar fashion to form a stacked hydrogen-bonded network with the cubane scaffold. When this nitro group is moved to the metaposition, as is the case in compound $\mathbf{3 1}$, the packing pattern is altered significantly. ${ }^{[16]}$ The $\mathrm{O}^{1}$ is now forming head-to-tail dimers with the cubane hydrogen atoms and this is augmented by $\mathrm{O}^{4}$ interacting with the cubane hydrogens from the opposite side of the cubane. The nitro groups can be seen to interact with both the cubane hydrogen on one oxygen and the benzene hydrogens on the other oxygen atom. This forms a stacked head-to-head network facilitated by the nitro group (Figure S10). The final compound in this section is one with the paranitrobenzloxy group on the 4-position of the cubane (32). ${ }^{[161]}$ The cubane hydrogen atoms are interacting with $\mathrm{O}^{1}$ in a head-tohead overlapped fashion with a secondary interaction seen between $\mathrm{O}^{2}$ and the benzene hydrogens (Figure S11). The nitro group in this structure appears to be less directive as seen with the similar structures of $\mathbf{3 0}$ and $\mathbf{3 1}$, as short contacts to this group can be seen forming towards to the methyl hydrogen atoms of the methyl ester.

When including larger ester groups (Figure 10, bond lengths and angles are shown in Table S4) it is noted that the cubane hydrogens are less likely to interact with the ester groups due to the increased competition of functionalized moieties within the cubane framework. Looking at the structure of $\mathbf{3 3}$ it can be seen that the cubane has now ceased forming hydrogen-bonded pairs with the ester. ${ }^{[16 i]}$ In fact, there appears to be a preference for $C_{-}$ $\mathrm{H} \cdots \mathrm{C}_{\text {cubane }}$ short contacts present rather than a typical hydrogenbond (Figure S12). Moving to the propyl ester (34), there is a return to the cubane interacting with $\mathrm{O}^{1}$ accepting a hydrogen from the cubane scaffold and $\mathrm{O}^{2}$ interacting with a $\mathrm{CH}_{2}$ of the propyl chain to form a network of hydrogen interactions (Figure 11A). ${ }^{[16 i]}$ In this structure, the presence of $\mathrm{C}-\mathrm{H} \cdots \mathrm{C}$ short contacts is also noted between the cubane scaffolds. For compound 35 , no hydrogen atoms were included in the submitted structural data, therefore no contacts can be deduced. ${ }^{[16 b]}$ Compound 36 shows the $\mathrm{CF}_{3}$ groups displaying some partial preference for halogen-bonding with the cubane scaffold, but the ester functionality is seen to form a dimer through $\mathrm{O}^{2 / 3}$ and the $\mathrm{CH}_{2}$ hydrogens (Figure 11B). ${ }^{[16 i]}$ The cubane unit appears to be held in a position where $\mathrm{C}$ $\mathrm{H}_{\text {cubane }} \cdots \mathrm{C}_{\text {cubane }}$ short contacts are present. In the structure of $\mathbf{3 7}$ this is changed due to the large bulk contributed by two $\mathrm{CF}_{3}$ moieties. In this case, there is a clear linear network created through the $\mathrm{O}^{2 / 3}$ interacting with the $\mathrm{CH}$ hydrogen atom (Figure S13). ${ }^{[16]}$ No close contacts appear around the cubane scaffold. The structure 38 shows a staggered linear network formed between the cubane scaffold and the terminal nitro groups in a reciprocated fashion (Figure S14). ${ }^{[16 i]}$ However, as no hydrogen atoms were provided in this structure no distances could be determined. The structure of $\mathbf{3 9}$ shows a linear network formed by the azide moiety yielding two close contacts. The first is the dimer formed between the $\mathrm{CH}_{2}$ hydrogens with the terminal nitrogen of the azide group. The second is the linear network formed between cubane hydrogen interacting nitrogen atom connected to the carbon skeleton (Figure S15). ${ }^{[16 e]}$

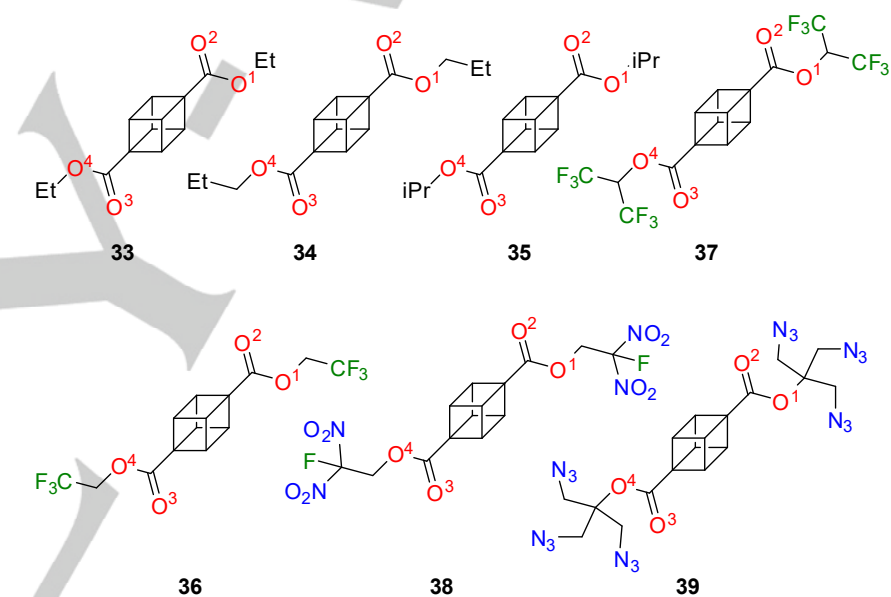

Figure 10. List of previously published extended ester 1,4-disubstituted cubane structures.

The final group of the esters features new compounds recently published by our group (40) ${ }^{[3 a]}$ and several determined as part of this work $(\mathbf{1 2}, \mathbf{4 1 - 4 4 )}$ (Figure 12, Table S5). The structure of $\mathbf{4 0}$ is an interesting example of what occurs when an extended rigid arm is present in the 4-position of a methyl ester cubane. The $\mathrm{O} \cdots \mathrm{H}$ interactions observed for compound $\mathbf{4 0}$ are similar to that of compound 24. The cubane hydrogen atoms interact with $\mathrm{O}^{1}$ in a dimer formation and $\mathrm{O}^{2}$ in a head-to-head overlapped fashion (Figure 13A). As discussed previously in the carboxylic acid section above, compound $\mathbf{1 2}$ results in the ester side of the molecule interacting with the cubane scaffold next layer forming a $\sim 90^{\circ}$ rotation between the layers of molecules. This is achieved through atom $\mathrm{O}^{2}$ and the cubane hydrogen atom (Figure 6). The structure of $\mathbf{4 1}$ shows more prevalent contacts between the methyl ether of the benzene ring and the cubane hydrogens. This type of interaction is aided by a halogen-bond between the methyl ether and the iodine of the cubane scaffold 
which facilitates overlapped dimers (Figure 13B and 13C). The structure of $\mathbf{4 1}$ shows that when sufficient competing groups are introduced to the cubane scaffold, the ester is less likely to form hydrogen-bonds with the cubane scaffold.

A)
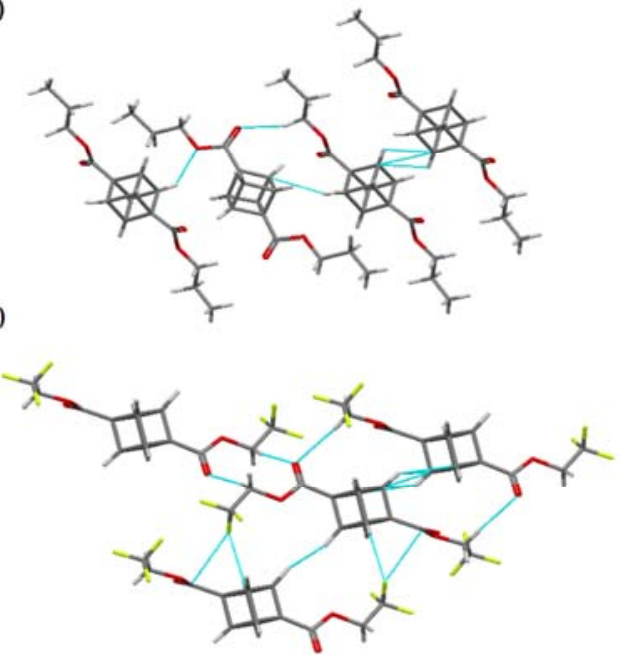

Figure 11. View of the molecular arrangement in the crystal of compound 34 (A) and 36 (B) represented as a stick model. Each view shows the preference for ester interactions within the given structure. Interactions are indicated by dashed blue lines.
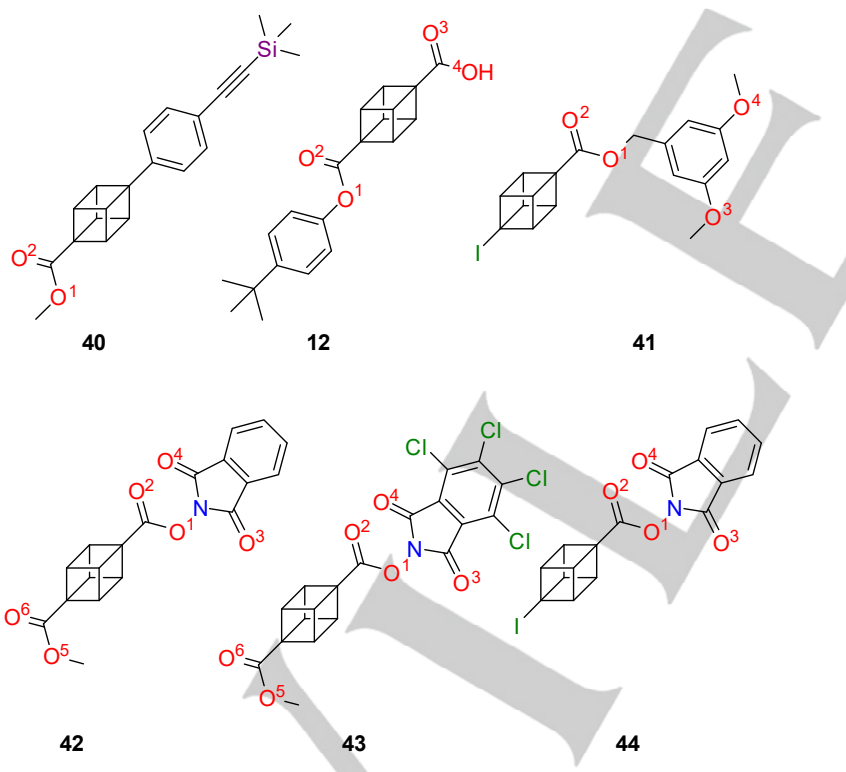

Figure 12. 1,4-Disubstituted cubane ester structures.

This leaves the final section of the esters series which concerns redox-active esters. These compounds were previously synthesized by our group as a method to functionalize the 4position of a cubane scaffold through a radical-mediated mechanism. ${ }^{[4 a]}$ Through this project, three such redox active esters were crystallized to a quality sufficient for X-ray diffraction analysis (42-44). The structure of $\mathbf{4 2}$ shows an interesting headto-tail overlapped network through the cubane hydrogen atoms interacting with $\mathrm{O}^{1}$ and $\mathrm{O}^{5} \cdot{ }^{[4 a]}$ This is stabilized though $\mathrm{O}^{2 \cdots \pi}$ and $\mathrm{O}^{6} \cdots \mathrm{H}-\mathrm{C}_{\mathrm{Me}}$ interactions (Figure $14 \mathrm{~A}$ and $14 \mathrm{~B}$ ). The cubane scaffold is also held at an ideal distance to partake in C$\mathrm{H}_{\text {cubane }} \cdots \mathrm{C}_{\text {cubane }}$ short contacts with the nearest cubane unit. Additionally, as seen in Figure $14 \mathrm{~A}$ and $14 \mathrm{~B}$, the additional oxygen atoms included in the redox active ester can be seen to interact with the cubane hydrogen atoms on one side (C$\mathrm{H}_{\text {cubane }} \cdots \mathrm{O}^{4}$ ) and the indoline hydrogens on the other side $\mathrm{C}-$ $\mathrm{H}_{1 \mathrm{n}} \cdots \mathrm{O}^{3}$ ) which results in an offset pattern of parallel sheets. When the redox active ester is substituted with chlorine atoms (43) a similar pattern emerges with cubane hydrogen atoms interacting with $\mathrm{O}^{1}$ and $\mathrm{O}^{5}$ to form the same head-to-tail overlap. The same $\mathrm{O}^{2 \cdots \pi} \mathrm{m}$ and $\mathrm{O}^{6} \cdots \mathrm{H}-\mathrm{C}_{\mathrm{Me}}$ connections are observed in this structure to form the stacked network. The main differences occur due to the chlorine atoms occupying the indoline moiety removing any hydrogen-bonding aspects. This results in a headto-tail overlapped structure due to hydrogen-bonds between $\mathrm{C}$ $\mathrm{H}_{\text {cubane }} \cdots \mathrm{O}^{3}, \mathrm{C}-\mathrm{H}_{\mathrm{Me}} \cdots \mathrm{O}^{4}$, and $\mathrm{C}-\mathrm{H}_{\text {cubane }} \cdots \mathrm{O}^{5}$ (Figure $14 \mathrm{C}$ and 14D). Also evident in this structure are several halogen-bonds between $\mathrm{C}-\mathrm{Cl} \cdots \mathrm{O}^{2}, \mathrm{C}-\mathrm{Cl} \cdots \mathrm{O}^{4}, \mathrm{C}-\mathrm{Cl} \cdots \mathrm{O}^{6}, \mathrm{C}-\mathrm{Cl} \cdots \mathrm{H}, \mathrm{C}-\mathrm{Cl} \cdots \mathrm{H}$, and $\mathrm{C}-\mathrm{Cl} \cdots \mathrm{Cl}$ which increase the space surrounding the benzene moiety resulting in an increase in the $b$ - and $c$-axis lengths by $0.5-1 \AA$, compared to compound 42 . When the methyl ester is replaced by an iodine atom (44) some unique changes occur. Specifically, in the stacking (Figure 14E and $14 \mathrm{~F}$ ), there is no head-to-tail overlap as seen in $\mathbf{4 2}$ or $\mathbf{4 3}$, due to the absence of the ester. Rather, there is quite a beautiful threefold contact sequence of hydrogen-bonding $\left(\mathrm{C}-\mathrm{H}_{\text {cubane }}{ }^{\cdots \mathrm{O}^{1}}\right)$, halogen $\left(\mathrm{C}-\mathrm{I} \cdots \mathrm{H}_{\text {cubane }}\right)$, and a close contact $\left(\mathrm{C}-\mathrm{O}^{2 \cdots \mathrm{O}^{3}}\right)$ which is repeated to form a neat stacking pattern. The secondary interactions of note are $\mathrm{C}-\mathrm{H}_{\text {cubane }} \cdots \mathrm{O}^{4}, \mathrm{C}-\mathrm{H}_{\mathrm{In}} \cdots \mathrm{O}^{4}, \mathrm{C}-$ $\mathrm{H}_{\text {cubane }} \cdots \mathrm{O}^{3}, \mathrm{C}-\mathrm{I} \cdots \mathrm{H}_{\mathrm{In}}, \mathrm{C}-\mathrm{I} \cdots \mathrm{H}_{\mathrm{ln}}$, and $\mathrm{C}-\mathrm{H}_{\mathrm{In}} \cdots \mathrm{C}_{\text {cubane }}$ which results in a wedge shape packing pattern.
A)

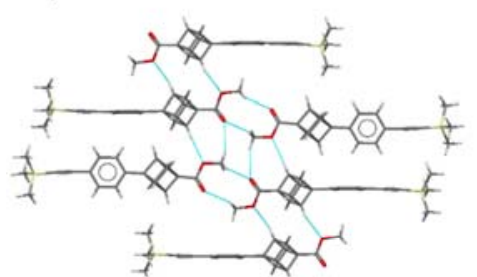

C)

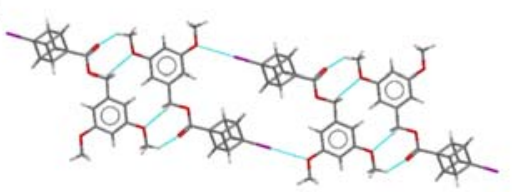

Figure 13. View of the molecular arrangement in the crystal of compound $\mathbf{4 0}$ (A) and 41 (B and $C$ ), represented as a stick model. Each view shows the preference for ester interactions within the given structure. Interactions are indicated by dashed blue lines. 
A)

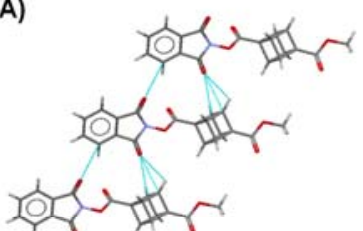

C)

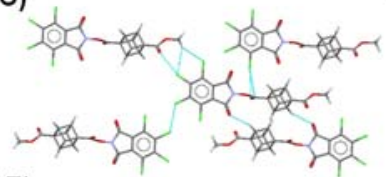

E)

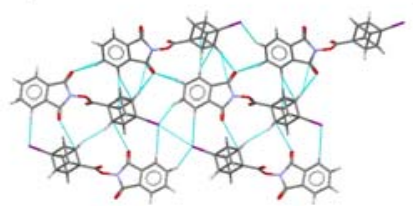

B)

D)
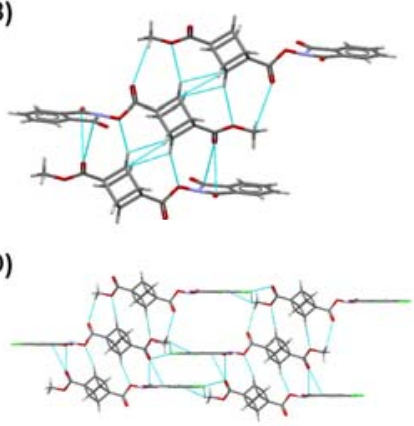

F)

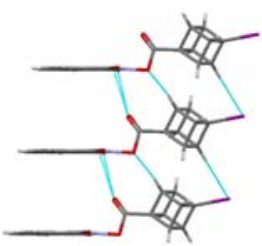

Figure 14. View of the molecular arrangement in the crystal of compound 42 ( $A$ and $B), \mathbf{4 3}(C$ and $D)$, and $\mathbf{4 4}(E$ and $F$ ) represented as a stick model. Each view shows the preference for ester interactions within the given structure. Interactions are indicated by dashed blue lines

\section{Halogens}

As of yet, there have been no studies into the effects that halogen-bonding has on the cubane hydrogen interactions.
Halogen contacts are one of the most common means, next to hydrogen-bonds, to achieve chemical directionality in a crystal structure. ${ }^{[18]}$ Some of these compounds have been covered in previous sections with regards to their second functionality (either methyl ester, carboxylic acid, or a carboxamide). This section shall focus mainly on how the cubane scaffold directly interacts with halogens atoms. Figure 15 shows all the published 4-halogeno-1-(R)-substituted cubane structures (where $R$ is a second functional group) and those determined as part of this work (bond lengths and angles are given in Table S6). In this section halogen-bonding between cubane moieties is quite apparent. Halogen-bonding, as described by the IUPAC standard occurs when there is evidence of a net attraction between the electrophilic region of associated with a halogen atom in a molecular entity and a nucleophilic region in another, or the same, molecular entity. ${ }^{[19]}$ Just like in hydrogen bonding, the bond lengths and angles are subject to the atom types involved in the interaction. ${ }^{[20]}$ However, there are two classes of halogen bonds type I (with an angle of $140-180^{\circ}$ ) and type II (with an angle of $90-120^{\circ}$ ) which can be used for a quick classification. ${ }^{[21]}$ Both type I and type II appear in the cubane structures below which suggests that these interactions are subject to close packing (type I) and electrostatic forces (type II). Cavallo et al. have written a comprehensive review of the nature of the halogen bond which is an excellent source for further information. ${ }^{[22]}$

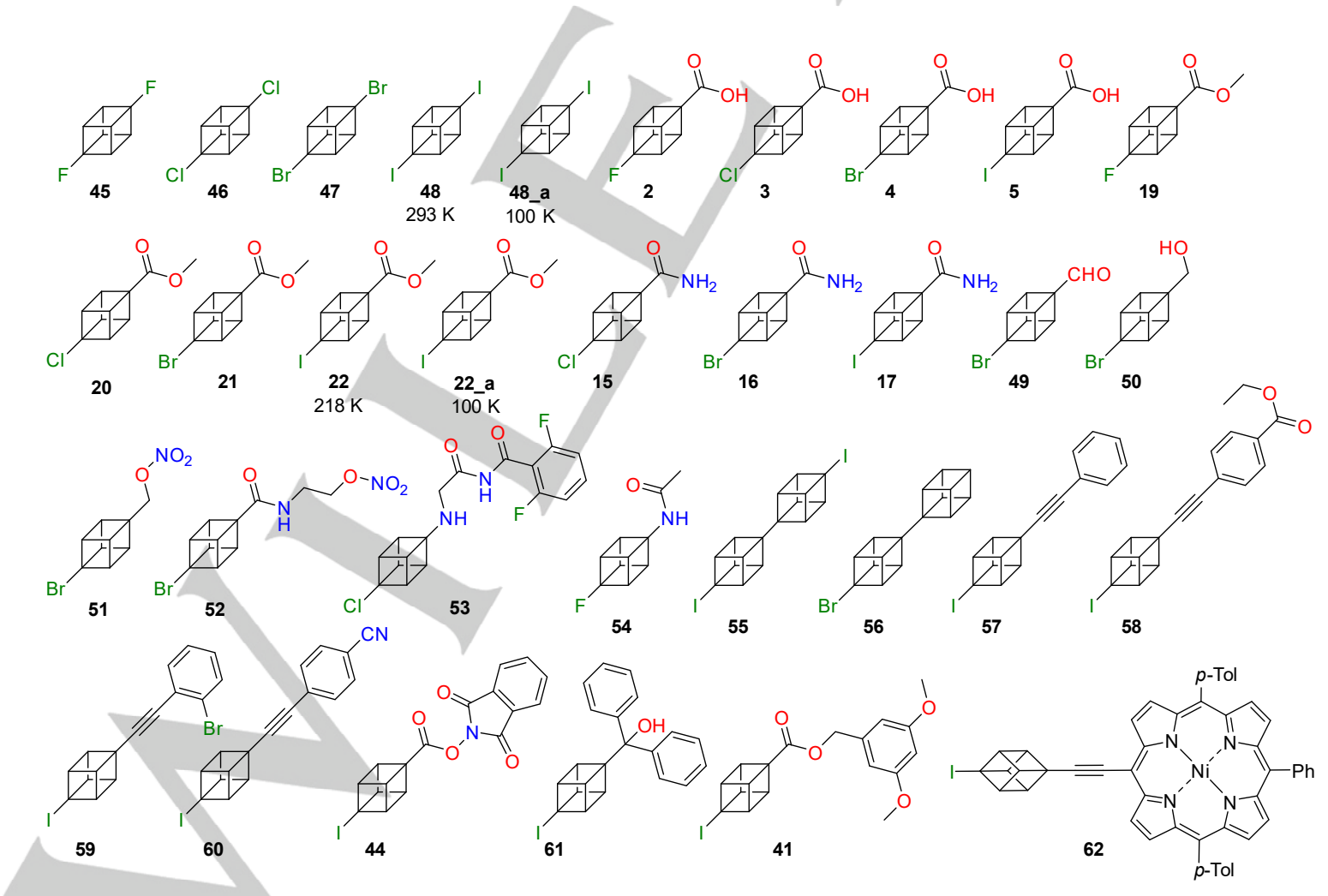

Figure 15. List of all 1,4-disubstituted cubane structures with a halogen attached to the 4-position. 
A)

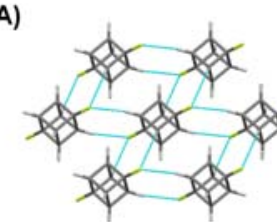

C)

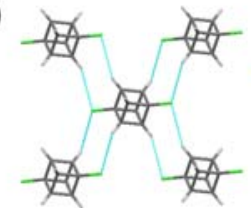

B)

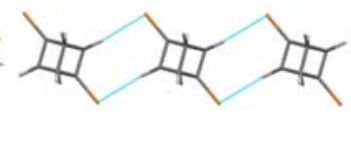

D)

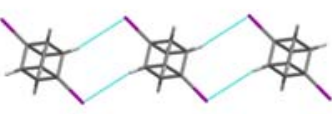

Figure 16. View of the molecular arrangement in the crystal of compound 45 (A), 47 (B), 46 (C), and 48 (D) represented as a stick model. Each view shows the preference for halogen interactions within the given structure. Interactions are indicated by dashed blue lines.

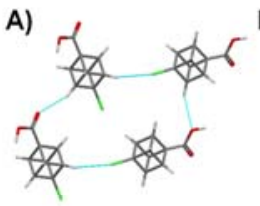

D)

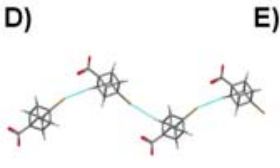

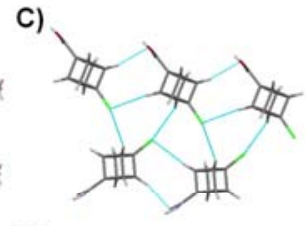

F)

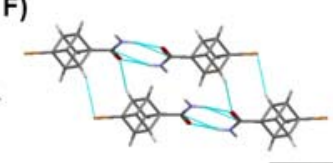

Figure 17. View of the molecular arrangement in the crystal of compound 3 $(A), 20(B), 15(C), 4(D), 21(E)$, and $16(F)$ represented as a stick model. Each view shows the preference for halogen interactions within the given structure. Interactions are indicated by dashed blue lines.

A)

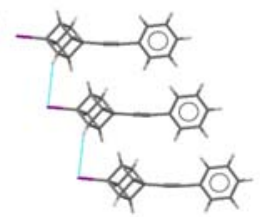

C)

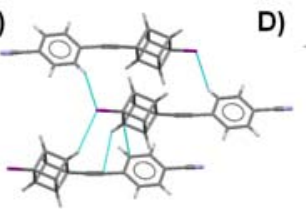

D)
B)
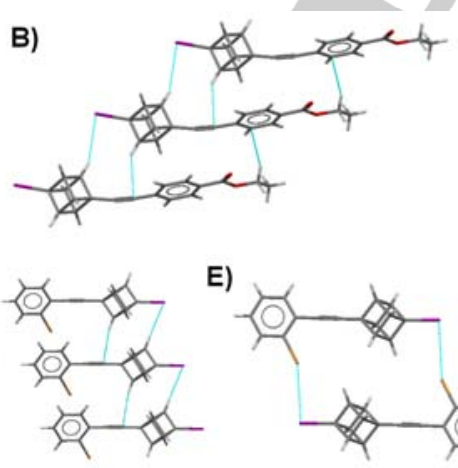

E)

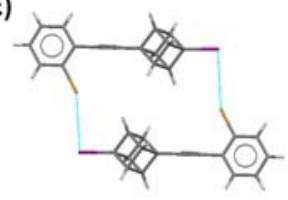

Figure 18. View of the molecular arrangement in the crystal of compound $\mathbf{5 7}$ (A), $58(B), 60(C)$, and 59 (D and E) represented as a stick model. Each view shows the preference for halogen interactions within the given structure. Interactions are indicated by dashed blue lines

The 1,4-dihalogenocubanes (45-48) show typical hydrogenhalogen interactions (Figure 16). ${ }^{[16 a, 23]}$ This is due to the fact that these are the only functional groups present in these structures. In this series, there is a correlation between atom size and the packing pattern observed. The difluoro cubane $\mathbf{4 5}$ shows a tighter packing, but also that the contacts are consistently bifurcated centering on the fluorine atom. The dichloro cubane 46 displays only short contacts and as a result, a much looser

packing pattern is observed. The dibromo cubane $\mathbf{4 7}$ and the diiodo derivative (48) form linear networks rather than cage like networks seen in $\mathbf{4 5}$ and $\mathbf{4 6}$. The structure of $\mathbf{4 8} \mathbf{a}$, which is a low temperature structure of $\mathbf{4 8}$ (48 originally determined at room temperature, 48_a determined at $100 \mathrm{~K}$ ). Both structures have the same unit cell and as a result, have the same short contacts present for compound $\mathbf{4 8}$ a as 48 with only a minor decrease in bond length (Figure S16).

When alternate functionalities are incorporated onto the 1position of the cubane scaffold, as seen with carboxylic acids (2-5), methyl esters (19-22), or primary carboxamides (15-17), the hydrogen-bond donor/acceptor groups form the primary interactions in these complexes with the halogen functionality secondary in terms of directing packing. ${ }^{[6-7,16 \mathrm{~h}]}$ In the carboxylic acid group, the 4-fluoro cubane (2) forms dimers, complementary to the head-to-head dimer of the acid, through an overlap of $\mathrm{H} \cdots \mathrm{F}$ contacts resulting in a staggered hydrogenbonding halogen-bonding system (Figure S17). The use of a larger halogen atom, such as chlorine, in compound 3 results in linking two catemer hydrogen-bonded networks with $\mathrm{H} \cdots \mathrm{Cl}$ interaction channels to form an alternate network of hydrogenbonding halogen-bonding system (Figure 17A).

Moving to bromine, the structure of $\mathbf{4}$ shows predominantly hydrogen-bonded networks in which the bromine atoms interact with the hydrogen on the cubane in the next layer rather than the cubane which is in the same layer as seen with $\mathbf{2}$ and $\mathbf{3}$ (Figure 17D). The structure of compound $\mathbf{5}$ is the first example where a direct halogen-halogen-bond is observed. The iodine atom interactions which allow for an alternating linear network of halogen and hydrogen contacts are periodically tethered together in layers by cubane hydrogen atoms interacting with the carboxylic acid (Figure S18). Unlike the carboxylic acids the esters do not have a hydrogen-bond donating ability and as a result, the ester moiety tends to stack with the cubane moiety in a dimer fashion. This forms two types of packing based on halogen size. Both the 4-fluoro (19) and 4-chloro derivatives (20) form tight networks where the esters can interact face-to-face. However, due to the size difference, fluorine interacts in a bifurcated fashion with the nearest hydrogen to the halogen substitute (Figure S19), whereas chlorine interacts with the hydrogens diagonal closest to the ester function group (Figure 17B). ${ }^{[6 \mathrm{~b}]}$ Both the 4-bromo (21) and the 4-iodo (22) compound show a more staggered and wider packing pattern. Due to the larger size of a halogen atom, the halogen interacts with the cubane in the above and below layers, as opposed to the inline contacts observed for compounds 19 and 20. Interestingly, the converse is observed with the bromine interacting with the hydrogen atoms closest to the ester function group (Figure 17E) and the iodine interacts with the nearest hydrogen to the halogen substitute (Figure S20_left), both in a bifurcated fashion. Additionally, when looking at the low-temperature structure 22_a there is a shift to more distance between the halogen side of the cubane scaffold with a direct $|\cdots|$ interaction now visible (Figure S20_right). The carboxamides cubanes show mainly dimer formation between the carboxamides moieties as mentioned above. This results in a head-to-head dimer between carboxamides leaving the halogen atoms to point towards each other. The 4-chloro derivative 15 shows two short $\mathrm{Cl} \cdots \mathrm{H}$ contacts which form an alternating network with layers of 
halogen-bonding and hydrogen-bonding interactions orthogonal to each other in a zig-zag pattern (Figure 17C). Moving to the 4bromo-cubane structure 16 a change to a stacked $\mathrm{Br} \cdots \mathrm{H}$ network is observed (Figure 17F). The 4-iodo-cubane structure 17 shows two short contacts with an $\mathrm{I} \cdots \mathrm{H}$ network similar to compound 15 and an $|\cdots|$ network. Together these form an alternating network with layers of halogen-bonding and hydrogen-bonding interactions (Figure S21). As seen in Figure 17, moving from carboxylic acid to methyl ester to carboxamide, there is a clear shift in preference to packing patterns which are not directed by the halogen, indicating that any connections seen are secondary to the hydrogen-bonding motif.

Several 4-bromo-cubane structures, with alternate substitution patterns and size of the substituents, have been deposited to the CCDC over the past few years. The structure of 4bromocubane-1-carbaldehyde (49) exhibits a linear stacking pattern with the cubane scaffolds in line with each other (Figure S22). ${ }^{[24]}$ Unfortunately, the structure is highly disordered, with the bromine and aldehyde being modeled over each other in multiple parts, and hydrogen atoms have not been assigned in the deposited structure. Therefore, this linear stacking can only indicate the potential interactions between the cubane scaffolds. The structure of 4-bromo-1-(hydroxymethyl)cubane (50) shows the alcohol moiety in a linear hydrogen-bonding fashion (Figure S23). ${ }^{25]}$ This allows the bromine atoms to interact with the hydrogen of the cubane in the next layer to create a 3D lattice. When substituting hydroxymethyl for a methyl nitrate (51) the $\mathrm{NO}_{2}$ group interacts with the cubane the $\mathrm{CH}_{2}$ hydrogen atoms. ${ }^{[12 a]}$ This leaves bromine atoms close to each other to form a direct halogen-bonded network (Figure S24). The structure of 52, 2-(4-bromocubane-1-carboxamido)ethyl nitrate, shows a linear hydrogen-bond network similar to that of the carboxamide 16, and a $\mathrm{Br} \cdots \mathrm{H}$ network that interacts with the cubanes hydrogen atoms in the next layer (Figure S25). ${ }^{[26]}$

$N$-(4-chlorocuban-1-yl)glycyl)-2,6-difluorobenzamide) (53) is probably the most functionalized 4-halocubane structure currently published (Figure S26). ${ }^{[3]}$ With an amine, carbonyl, and halogen functional groups available competition for the cubane hydrogen atoms is high. A bifurcated interaction is noted centering on the chlorine atom with a cubane hydrogen and a benzene hydrogen atom. Other noticeable cubane contacts are between the fluorine atoms of the benzene ring and the oxygen of the carbonyl furthest from the cubane, both of which interact with the cubane in a bifurcated fashion. Additionally, the second fluorine atoms interact with a cubane hydrogen atom on the opposite side. The remaining interactions are a plethora of dimers formed between the amide type linkers through classical hydrogen-bonding.

Compound $\mathbf{5 4}$ contains an acetamide on one side and a fluorine on the other. ${ }^{[16 a]}$ This structure shows the similar bifurcated contact between fluorine and hydrogen atoms as seen in compound 19 and 2. This is in addition to the $\mathrm{N}-\mathrm{H} \cdots \mathrm{O}$ interactions, which are rotated at $90^{\circ}$ to each other, resulting in an alternating packing of hydrogen-bonding and halogenbonding partners (Figure S27).

The next two cubane structures are directly linked cubane scaffolds with halogen substituents $(55$ and 56$) .{ }^{[27]}$ In these structures, rather than the halogen-hydrogen contacts, the nonclassical hydrogen-bond between cubane scaffolds is much more common. This is due to the second cubane scaffold included in these structures which allows for the free cubane sides to interact more with each other. Compound $\mathbf{5 5}$ is a prime example of this, as even though two halogens are present, there are no connections between them and any cubane $\mathrm{H}$-atoms. This results in a highly ordered system where the halogens are held between layers of cubanes but do not interact with the cubane scaffold directly (Figure S28). On the other hand, the mono-halogenated compound $\mathbf{5 6}$ shows that the bromine atoms will interact with hydrogen atoms due to a tighter packing as a result of the less symmetric molecule (Figure S29).

A)

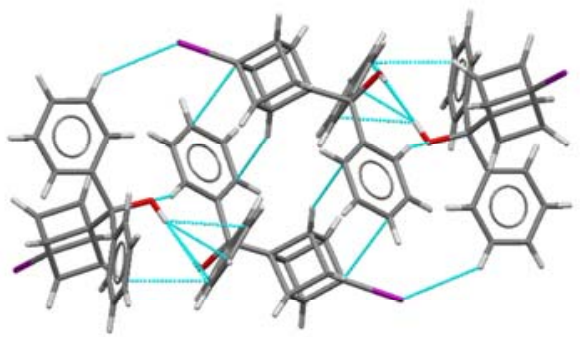

B)

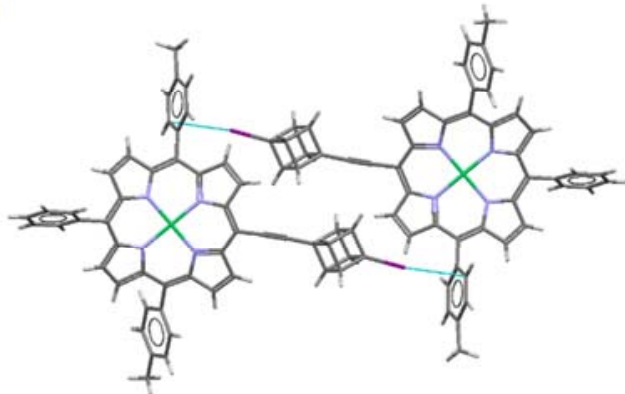

Figure 19. View of the molecular arrangement in the crystal of compound $\mathbf{6 1}$ (A) and 62 (B) represented as a stick model. Each view shows the preference for halogen interactions within the given structure. Interactions are indicated by dashed blue lines.

Compounds 57-60 vary only by the substitution of the benzene ring. Using compound $\mathbf{5 7}$ as a base model for this set highlights two $\mathrm{C}-\mathrm{H}_{\text {cubane }} \cdots$ interactions that aid in lateral stacking and one $\mathrm{C}-\mathrm{H}_{\text {cubane }}{ }^{\cdots} \pi$ contact that allows for vertical stacking (Figure 18A). The inclusion of the ethyl ester, $\mathbf{5 8}$ shows the addition of a C- $\mathrm{H}_{\text {cubane }} \cdots \pi$ interaction with the ethynyl moiety to stabilize a $\mathrm{C}$ $\mathrm{H}_{\text {cubane }} \cdots \mid$ lateral network (Figure 18B). This is in addition to the ester cubane connections which are mainly involved in a headto-tail overlapped vertical stack (Figure S30). Upon the inclusion of a cyano group to the para-position of the benzene ring (60), two $\mathrm{l} \cdots \mathrm{H}$ networks are seen between the halogen and cubane hydrogen atoms in a stacking type pattern and the halogen and aromatic hydrogen atoms in a head-to-tail dimer fashion (Figure $18 \mathrm{C})$. This is aided by two cubane hydrogen atoms interacting with the ethynyl- $\pi$ system, which seems typical for these types of compounds. Additionally, there is a hydrogen-bonding contact between cyano groups and the aromatic rings in a bifurcated fashion due to the space is taken up by the iodine atoms. This results in a head-to-head dimer and an orthogonal interaction between another pair of molecules to form a $2 \times 2$ layering crossover pattern (Figure S31). The 2-bromobenzene derivative 59 shows a similar $\mathrm{I} \cdots \mathrm{H}$ and $\mathrm{C}-\mathrm{H}_{\text {cubane }} \cdots \pi$ network common for 
this series. However, the main feature is the direct $\mathrm{I} \cdots \mathrm{Br}$ halogen-bond to create a head-to-tail dimer between the molecules (Figure 18D and 18E).

The two halo-ester cubane structures 44 and 41, previously covered in the ester section, show the effect of including a halogen atom. The structure of $\mathbf{4 4}$ shows one contact between the cubane hydrogen and the iodine atoms in a similar fashion seen in the structures of 57-60. Coupled with the ester interaction there are several $\mathrm{l} \cdots \mathrm{H}$ short contacts between the indoline hydrogen atoms. Of most interest is the direct $|\cdots|$ halogen-bond in a type I manner forming a head-to-head contact (Figure 14E and 12F). The structure of $\mathbf{4 1}$ is much simpler with the only cubane contact involved with the methyl ester of the indoline ring. The only noticeable halogen interaction is between the iodine and the oxygen of the methyl ester (Figure 13C).

The final two 4-halogeno-cubane structures are possibly the most unique structures available. The first, 61, shows the inclusion of a highly bulky substitute around the cubane scaffold. There are clear short contacts between the cubane hydrogen atoms and the phenyl $\pi$-ring in a dimer fashion as seen in Figure 19A. The O-H shows short contacts towards the phenyl m-ring. The only halogen interaction is seen between the phenyl hydrogen atoms creating a linear network. This shows the noticeable effects a large group can cause in shielding the cubane scaffold. The final structure, 62, has the largest substituent attached to a cubane scaffold. ${ }^{[4 a]}$ The addition of a porphyrin molecule has an interesting effect on the cubane structure. ${ }^{[28]}$ The tolyl substituents of the porphyrin 'sandwich' the cubane scaffold and it is tethered with a $\mathrm{C}-\mid \cdots \pi$ to form a tightly packed structure (Figure 19B).

By looking at the connections and packing of the 4-halo-1-(R)cubane structures it is clear, as is the case from acid, ester, or carboxamide cubane derivatives, that there are a substantial number of halogen $\cdots$ hydrogen interactions with few halogen $\cdots$ halogen contacts present in most structures. However in these structures, the halogen contacts are less directive than the acid, ester, or carboxamide interactions. This is clearly seen in Figure 17, with the three alternate functionalities causing three different packing patterns. When moving to the rod-like structures (Figure 18), the most common motif is the $\mathrm{C}-\mathrm{l} \cdots \mathrm{H}$ connection between the cubane scaffolds. This is typically stabilized with a C- $\mathrm{H}_{\text {cubane }} \cdots \mathrm{C}_{\text {ethynyl }}$ interaction. The only structure which differs from this packing order is compound $\mathbf{6 0}$ where the $-\mathrm{CN}$ group results in a detailed $\mathrm{C} \equiv \mathrm{N} \cdots \mathrm{C}_{\mathrm{Bz}}$ network which is rotated, leaving more space in the crystal packing for contacts between $\mathrm{C}-\mathrm{l} \cdots \mathrm{H}$ of the iodine group and benzene hydrogens (Figure S31). These results indicate the importance of the cubane hydrogen and halogen atoms.

\section{Acetylene moieties}

For compounds $\mathbf{4 0}, \mathbf{5 7}-\mathbf{6 0}$, and $\mathbf{6 2}$ it is noted that there is a high frequency of ethylene moieties interacting with the cubane scaffold in a type of non-classical hydrogen-bond. This prompted further investigations to see if this feature was shared by other cubane structures with a similar moiety. In the literature, there are only five examples. This section will focus mainly on the connections between the ethylene moiety and the cubane hydrogen atoms, with alternate functionalities and the resultant interactions already covered in the previous section, unless the structure only appears in this section. Figure 20 shows the ethynyl bearing cubane scaffolds from the literature and six structures determined as part of this work. Bond lengths and angles are shown in Table S7.
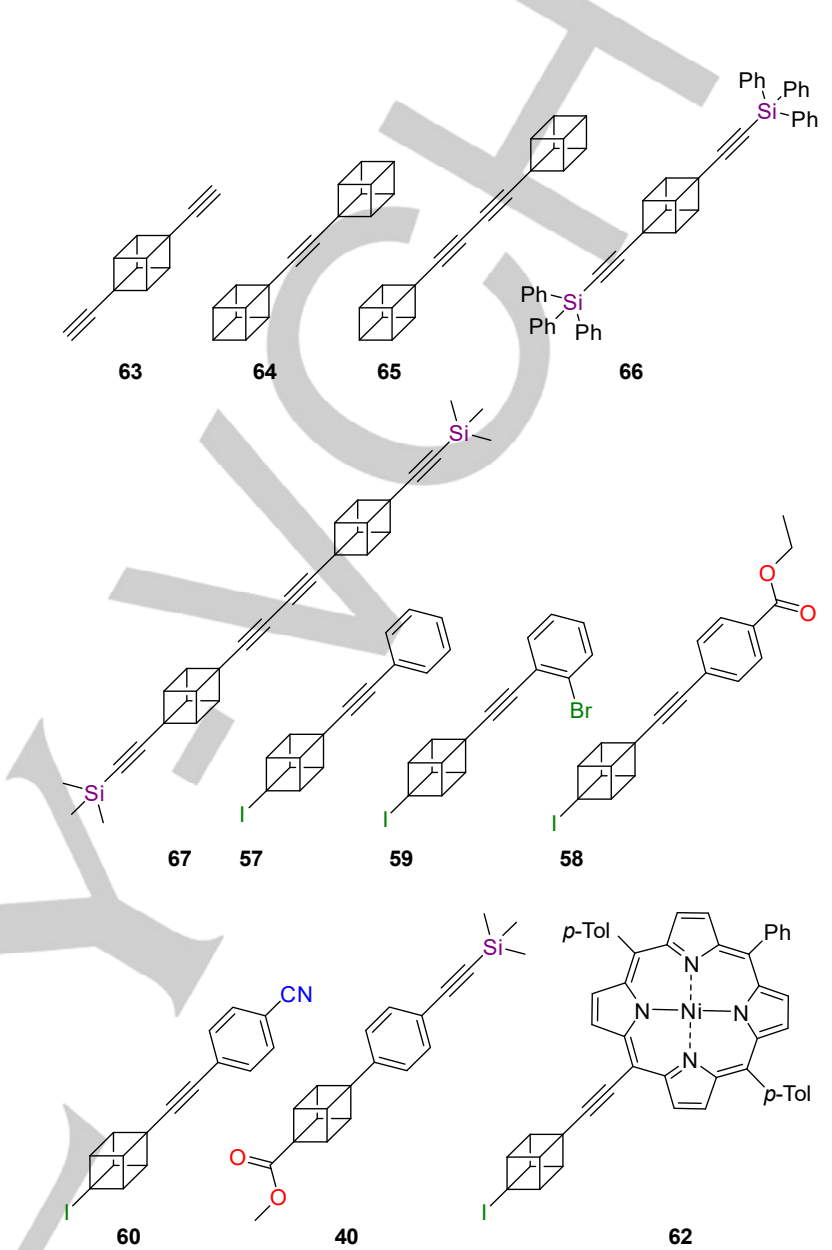

Figure 20. Ethynyl bearing 1,4-disubstituted cubane structures.

The structure of $\mathbf{6 3}$ is the simplest and the most characteristic of the ones provided for the $\mathrm{C}-\mathrm{H}_{\text {cubane }} \cdots \mathrm{C}_{\text {ethynyl }}$ interactions under consideration. ${ }^{[29]}$ Due to its small size and simple structure it is, quite literally, surrounded by the non-classical hydrogen-bonds that make up the subject of this section. This is mainly seen in the form of $\mathrm{C}-\mathrm{H}_{\text {ethynyl }} \cdots \mathrm{C}_{\text {ethynyl }}$ interactions on one side of the triple bond with a complimentary $\mathrm{C}-\mathrm{H}_{\text {cubane }} \cdots \mathrm{C}_{\text {ethynyl }}$ contact on the other to form a herringbone network (Figure 21A). This holds each individual molecule in perfect alignment in a highly ordered system. When this structure is inverted, as is the case for $\mathbf{6 4}$, no $\mathrm{C}-\mathrm{H}_{\text {ethynyl }}{ }^{\cdots} \mathrm{C}_{\text {ethynyl }}$ motif can possibly form. The structure is then comprised of multiple $\mathrm{C}-\mathrm{H}_{\text {cubane }}{ }^{\cdots} \mathrm{C}_{\text {ethynyl }}$ connections in a similar fashion to 63; however, in a much more staggered linear network, both vertically and laterally (Figure 21B). ${ }^{[29]}$ When this linker is extended between two cubane scaffolds (65), the extra spacer between the two cubane scaffolds allows the cubane itself to occupy a space that is otherwise too small.[29] This results in, not only the $\mathrm{C}-\mathrm{H}_{\text {cubane }}{ }^{\cdots} \mathrm{C}_{\text {ethynyl }}$ contacts associated with this family of compounds, but now $\mathrm{C}-\mathrm{H}_{\text {cubane }} \cdots \mathrm{C}_{\text {cubane }}$ interactions, which previously were disfavored due to space constraints. This structure forms an interesting pattern of layers 
of molecules staggered at roughly $45^{\circ}$ to each other as shown in Figure $21 \mathrm{C}$. Compound 66 shows what happens by increasing the size of the substituents around the cubane scaffold. ${ }^{[29]}$ In direct contrast to 63 , there are no $\mathrm{C}-\mathrm{H}_{\text {cubane }} \cdots \mathrm{C}_{\text {ethynyl }}$ bonds. Rather, every contact is taken up by the phenyl groups in a $\mathrm{C}$ $\mathrm{H}_{\mathrm{Ph}} \cdots \mathrm{C}_{\text {ethynyl }}$ or $\mathrm{C}-\mathrm{H}_{\mathrm{Ph}} \cdots \mathrm{C}_{\text {cubane }}$ fashion (Figure S32). This indicates that low steric hindrances favor $\mathrm{C}-\mathrm{H}_{\text {cubane }} \cdots \mathrm{C}_{\text {ethynyl }}$ motifs, while large bulk inhibits such interactions. This is exemplified in the structure of $\mathbf{6 7}$ which is a combination of $\mathbf{6 6}$ and $65 .{ }^{[30]}$ In this structure $\mathrm{C}-\mathrm{H}_{\text {cubane }} \cdots \mathrm{C}_{\text {ethynyl }}$ connections return as the main linkage between molecules; however, due to the comparatively smaller but still significantly bulky trimethylsilyl group, no $\mathrm{C}-\mathrm{H}_{\text {cubane }}{ }^{\cdots} \mathrm{C}_{\text {cubane }}$ interactions are observed, forming a relatively ordered packing (Figure S33). Of the structures to be included in this section, this $\mathrm{C}-\mathrm{H}_{\text {cubane }}{ }^{\cdots} \mathrm{C}_{\text {ethynyl }}$ contact is quite common, except for compound $\mathbf{5 7 ^ { [ 4 a ] }}$ which has no noticeable connections.
A)

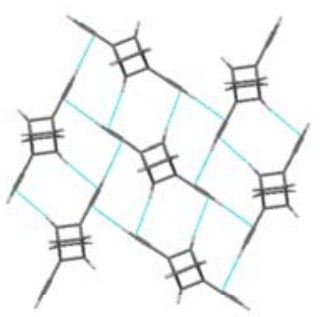

B)

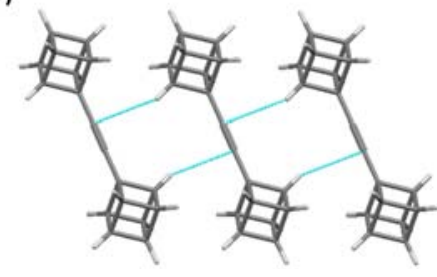

C)

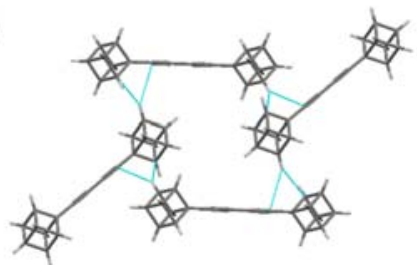

makes the $\mathrm{C}-\mathrm{H}_{\text {cubane }}{ }^{\cdots} \mathrm{C}_{\text {ethynyl }}$ partially directive, but the alternate functional group or bulk of the alternate group has a more significant effect on the packing pattern.
A)

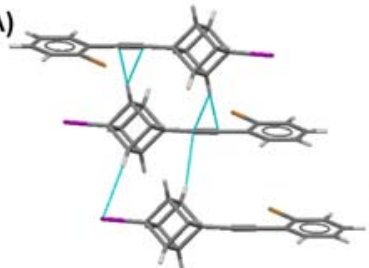

C)

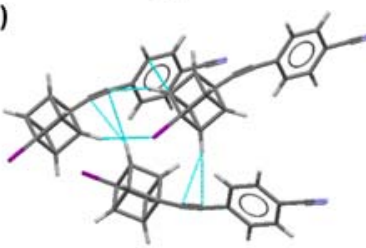

B)

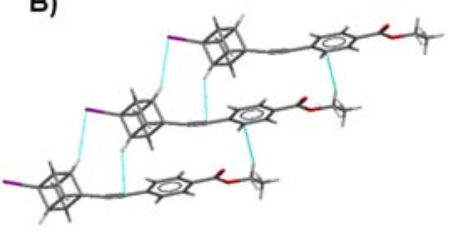

D)

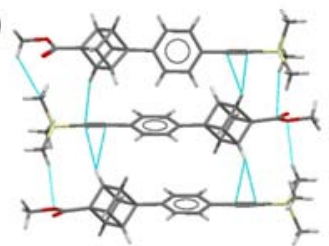

E)

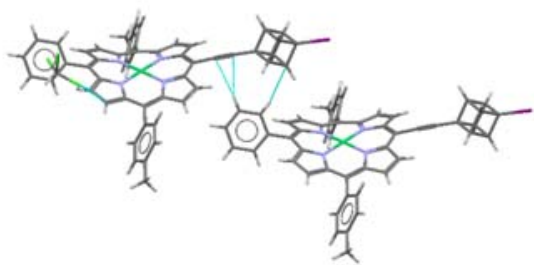

Figure 22. View of the molecular arrangement in the crystal of compounds of $59(A), 58(B), 60(C), 40(D)$, and $62(E)$ represented as a stick model. Each view shows the preference for $\mathrm{C}-\mathrm{H}_{\text {cubane }} \cdots \mathrm{C}_{\text {ethynyl }}$ interactions within the given structure. Interactions are indicated by dashed blue lines.

\section{Other 1,4-substituted cubanes}

The remaining 1,4-disubstituted cubane structures published in the literature that either did not fit into the above categories or are too limited in number have been covered in detail on page 14 of the supporting information.

Figure 21. View of the molecular arrangement in the crystal of compound (A), $64(B)$, and $65(\mathrm{C})$ represented as a stick model. Each view shows the preference for $\mathrm{C}-\mathrm{H}_{\text {cubane }} \cdots \mathrm{C}_{\text {ethynyl }}$ interactions within the given structure. Interactions are indicated by dashed blue lines.

The structure of $\mathbf{5 9}$ has an interesting network of C$\mathrm{H}_{\text {cubane }} \cdots \mathrm{C}_{\text {ethynyl }}$ contacts that accommodate a head-to-head overlap in one direction and a head-to-tail interaction in the other to form a repeating quartet throughout the crystal. This is aided by the $\mathrm{I} \cdots \mathrm{H}$ and $\mathrm{l} \cdots \mathrm{Br}$ contacts previously mentioned in the halogen section above (Figure 22A). The structure of $\mathbf{5 8}$ exhibits an overlapped network of $\mathrm{C}-\mathrm{H}_{\text {cubane }} \cdots \mathrm{C}_{\text {ethynyl }}$ connections which form the head-to-head structure, aided by $\mathrm{I} \cdots \mathrm{H}$ contacts. The ester in this structure plays a nominal role in forming head-to-tail dimers but has no interaction visible with the ethynyl group (Figure 22B). The structure of $\mathbf{6 0}$ shows $\mathrm{C}-\mathrm{H}_{\text {cubane }} \cdots \mathrm{C}_{\text {ethynyl }}$ contacts in a trimer formation. This is aided by the $\mathrm{I} \cdots \mathrm{H}$ bonds, as seen in both 59 and $\mathbf{5 8}$, and the roughly $70^{\circ}$ rotation of one of the contributing molecules (Figure 22C). The structure of $\mathbf{4 0}$, even though it does not have the same base motif, shows favorable $\mathrm{C}-\mathrm{H}_{\text {cubane }} \cdots \mathrm{C}_{\text {ethynyl }}$ contacts as a result of head-to-tail stacked network (Figure 22D). The final structure, 62, shows no $\mathrm{C}-\mathrm{H}_{\text {cubane }} \cdots \mathrm{C}_{\text {ethynyl }}$ interactions, preferring to form $\mathrm{C}-\mathrm{H}_{\mathrm{Ph}} \cdots \mathrm{C}_{\text {ethynyl }}$ interactions (Figure 22E). By looking at these structures $(\mathbf{4 0}, \mathbf{5 7}-$ $60,62-67$ ) there is a clear preference for the formation of $\mathrm{C}$ $\mathrm{H}_{\text {cubane }} \cdots \mathrm{C}_{\text {ethynyl }}$ regardless of the alternate functionality. This

\section{Conclusions}

In conclusion, we have investigated the structure of 1,4substituted cubane scaffolds with regards to all potential interactions (Figure 23). With this, we have highlighted the contacts between esters, halogens, ethynyl, nitrogen bearing groups and all remaining 1,4-substituted cubane structures existing in the literature, which has built on the previous work by Desiraju and co-workers on the interactions between cubane hydrogen atoms and acid or carboxamide moieties. This has revealed many interesting points, not only the contacts of the previously reported carboxylic acids and carboxamides, but also a plethora of connections are seen between the cubane scaffold and other heteroatoms groups. Interestingly the non-classical hydrogen-bonding between the cubane $\mathrm{H}$-atoms and other moieties such as another cubane scaffold or ethynyl groups appears to be just as directive as compared to the more classical hydrogen-bonding systems seen in the works of Desiraju.

We have also highlighted the fact that halogen atoms, while they do change the packing motif, appear to be secondary in 
directing the packing regarding other hydrogen-bonding groups such as carboxylic acids, esters, or carboxamides. Additionally, the halogen functionalized cubane structures appear to favor halogen-hydrogen contacts above that of halogen-halogen interactions with only a handful of structures appearing to have any contribution to the latter. Ester functionalized cubane structures appear to form similar types of catemer formation as seen with the carboxylic acids previously studied. As esters are exclusively hydrogen-bond acceptors, this is complementary to the hydrogen-bond donating ability of the cubane hydrogen atoms. In short, cubane is structurally close in size to a benzene ring along the 1,4-axis. However, the higher number of hydrogen atoms and the directions in which they are aligned is quite different to a benzene or a phenyl ring. This results in a multidirectional hydrogen-bond donor system which shows a larger potential of interactions possible and alternate packing systems. These packing systems are altered by the direction in which the hydrogens point as shown in the case study with the carboxylic acids. This demonstrates that the hydrogen atoms of the cubane may play much more of a role than previously discussed a facet which should be also considered when substituting cubane as an isostere for benzene or phenyl rings.

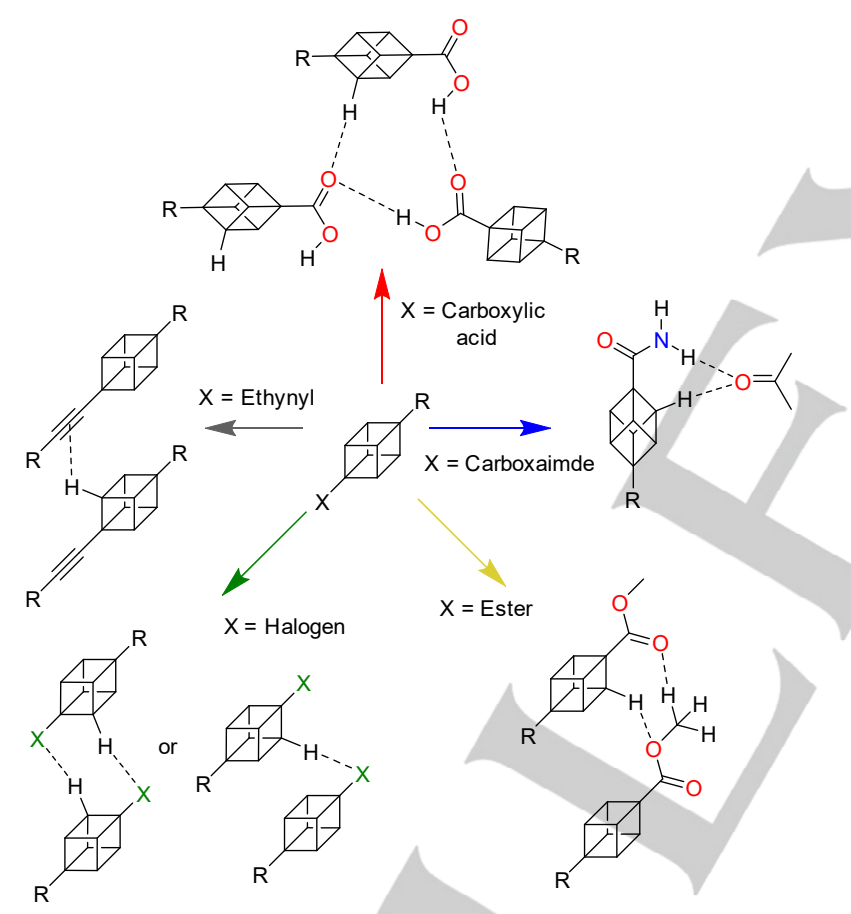

Figure 23. Summary image of the 1,4-disubstituted cubane structure interactions.

This work was aimed at an initial investigation into the interaction profile of cubane to better understand which moieties and substituent pattern will give rise to specific patterns. The next steps to this investigation are to combine the knowledge gained here and compare the cubane structures directly with all their benzene counter-parts. This would allow for a direct comparison to the differences and similarities seen in both cubanes and benzene interaction profiles. Following this, there are additional small molecule scaffold systems such as bicyclo[1.1.1]pentane, which can be added as components in further structural studies in the future. ${ }^{[4 b]}$ There are many potential avenues to expand this study in the future. The first of these is to cross reference the structures herein with that of neutron diffraction studies to obtain super fine structural details of the position of the cubane hydrogen atoms and their interactions. Additional studies comparing the bond strength to a bond distance of the interactions outlined here would provide more understanding of the nature of cubane hydrogen interactions included in this current study.

\section{Experimental Section}

All published data was obtained from the CCDC using the program ConQuest version 1.23 (update August 2018). ${ }^{[31]}$ All bond angles and bond lengths were measured using the program Mercury CSD version 3.10.2. ${ }^{[32]}$ All images in the manuscript were prepared by using Mercury CSD version 3.10.2. Thermal ellipsoid plots are included for all structures determined as part of this publication in the supporting information and were prepared using Olex2. ${ }^{[33]}$

Crystals were grown following the protocol developed by Hope by dissolving the compounds in either $\mathrm{DCM}$ or a $\mathrm{DCM} / \mathrm{MeOH}$ mixture and allowing for slow evaporation over time. ${ }^{[34]}$ Single crystal X-ray diffraction data for all compounds were collected on a Bruker APEX 2 DUO CCD diffractometer by using graphite-monochromated $\operatorname{MoK}_{\alpha}(\lambda=0.71073 \AA)$ radiation and Incoatec I $\mu \mathrm{S} \mathrm{CuK}_{\alpha}(\lambda=1.54178 \AA)$ radiation. Crystals were mounted on a MiTeGen MicroMount and collected at $100(2) \mathrm{K}$ by using an Oxford Cryosystems Cobra low-temperature device. Data were collected by using omega and phi scans and were corrected for Lorentz and polarization effects by using the APEX software suite. ${ }^{[35]}$ Using Olex2, the structure was solved with the $\mathrm{XT}$ structure solution program, using the intrinsic phasing solution method and refined against $\left|F^{2}\right|$ with $X L$ using least squares minimization. ${ }^{[33,36]}$ Hydrogen atoms were generally placed in geometrically calculated positions and refined using a riding model. Details of data refinements can be found in Table S10-S13 and further experimental and refinement detail can be found in the SI. CCDC1887763-1887774 contains the supplementary crystallographic data for this paper. These data sets can be obtained free of charge from The Cambridge Crystallographic Data Centre via http://www.ccdc.cam.ac.uk/data_request/cif.

Keywords: Cubane • non-covalent interactions • hydrogenbonding • halogen-bonding • non-classical hydrogen-bonding

\section{Acknowledgements}

This work was supported by a grant from Science Foundation Ireland (SFI IvP 13/IA/1894).

\section{Conflicts of interest}

The authors declare no conflict of interest.

\section{References}

[1] P. E. Eaton, Angew. Chem. Int. Ed. 1992, 31, 1421-1436.

[2] a) L. T. Eremenko, L. B. Romanova, M. E. Ivanova, D. A. Nesterenko, V. S. Malygina, A. B. Ermeev, G. V. Lagodzinskaya, V. P. Lodygina, Russ. Chem. Bull. 1998, 47, 1137-1140; b) H. Gunosewoyo, J. L. Guo, M. R. Bennett, M. J. Coster, M. Kassiou, Bioorg. Med. Chem. Lett. 2008, 18, 3720-3723; c) R. Pellicciari, G. Costantino, E. Giovagnoni, L. Mattoli, I. Brabet, J.-P. Pin, Bioorg. Med. Chem. Lett. 1998, 8, 1569-1574; d) A. S. Sklyarova, V. N. 
Rodionov, C G Parsons, G Quack, P. R Schreiner, A A. Fokin, Med. Chem. Res. 2013, 22, 360-366; e) S. M. Wilkinson, H. Gunosewoyo, M. L. Barron, A. Boucher, M. McDonnell, P. Turner D. E. Morrison, M. R. Bennett, I. S. McGregor, L. M. Rendina, M Kassiou, ACS Chem. Neurosci. 2014, 5, 335-339.

[3] a) B. A. Chalmers, H. Xing, S. Houston, C. Clark, S. Ghassabian, A. Kuo, B. Cao, A. Reitsma, C.-E. P. Murray, J. E. Stok, G. M Boyle, C. J. Pierce, S. W. Littler, D. A. Winkler, P. V. Bernhardt, C Pasay, J. J. De Voss, J. McCarthy, P. G. Parsons, G. H. Walter, M. T. Smith, H. M. Cooper, S. K. Nilsson, J. Tsanaktsidis, G. P. Savage, C. M. Williams, Angew. Chem. Int. Ed. 2016, 55, 35803585 ; b) B. A. Chalmers, H. Xing, S. Houston, C. Clark, S. Ghassabian, A. Kuo, B. Cao, A. Reitsma, C.-E. P. Murray, J. E. Stok, G. M. Boyle, C. J. Pierce, S. W. Littler, D. A. Winkler, P. V. Bernhardt, C. Pasay, J. J. De Voss, J. McCarthy, P. G. Parsons, G. H. Walter, M. T. Smith, H. M. Cooper, S. K. Nilsson, J. Tsanaktsidis, G. P. Savage, C. M. Williams, Angew. Chem. Int. Ed. 2018, 57, 8359-8359.

[4] a) S. S. R. Bernhard, G. M. Locke, S. Plunkett, A. Meindl, K. J. Flanagan, M. O. Senge, Chem. Eur. J. 2018, 24, 1026-1030; b) G M. Locke, S. S. R. Bernhard, M. O. Senge, Chem. Eur. J. 2018 Accepted Manuscript, DOI: 10.1002/chem.201804225.

[5] D. Das, G. R. Desiraju, Chem. Asian J. 2006, 1, 231-244

[6] a) S. S. Kuduva, D. C. Craig, A. Nangia, G. R. Desiraju, J. Am Chem. Soc. 1999, 121, 1936-1944; b) D. Das, R. K. R. Jetti, R. Boese, G. R. Desiraju, Cryst. Growth Des. 2003, 3, 675-681.

[7] S. S. Kuduva, D. Bläser, R. Boese, G. R. Desiraju, J. Org. Chem 2001, 66, 1621-1626

[8] J. Echeverría, G. Aullón, D. Danovich, S. Shaik, S. Alvarez, Nat Chem. 2011, 3, 323-330.

[9] a) P. K. Mykhailiuk, Org. Biomol. Chem. 2019, Accepted Manuscript, DOI: 10.1039/C1038OB02812E; b) Y. P. Auberson, C. Brocklehurst, M. Furegati, T. C. Fessard, G. Koch, A. Decker, L. La Vecchia, E. Briard, ChemMedChem 2017, 12, 590-598; c) T. A Reekie, C. M. Williams, L. M. Rendina, M. Kassiou, J. Med. Chem. 2018 Accepted Manuscript, DOl: 10.1021/acs.jmedchem.1028b00888; d) T. P. Stockdale, C. M Williams, Chem. Soc. Rev. 2015, 44, 7737-7763.

[10] T. Y. Luh, L. M. Stock, J. Am. Chem. Soc. 1974, 96, 3712-3713.

[11] a) C. L. Nygren, C. C. Wilson, J. F. C. Turner, The Journal of Physical Chemistry A 2005, 109, 2586-2593; b) M. Kubota, S. Ohba, Acta Cryst. Sect. B 1992, 48, 849-854; c) G. Bruno, L. Randaccio, Acta Cryst. Sect. B 1980, 36, 1711-1712; d) R. S Miller, I. C. Paul, D. Y. Curtin, J. Am. Chem. Soc. 1974, 96, 63346339; e) O. Kenji, K. Setsuo, H. Masao, Bull. Chem. Soc. Jpn. 1972, 45, 2651-2652.

[12] a) L. B. Romanova, L. S. Barinova, V. V. Zakharov, L. T Eremenko, G. G. Aleksandrov, I. L. Eremenko, Russ. Chem. Bull. 2010, 59, 1051-1055; b) O. Ermer, J. Lex, Angew. Chem. Int. Ed. $1987,26,447-449$.

G. R. Desiraju, Angew. Chem. Int. Ed. 2011, 50, 52-59.

[14] T. Steiner, Crystallogr. Rev. 2003, 9, 177-228.

[15] R. J. Doedens, P. E. Eaton, E. B. Fleischer, Eur. J. Org. Chem 2017, 2017, 2627-2630.

[16] a) H. Irngartinger, S. Strack, F. Gredel, A. Dreuw, W. Della Ernest, Eur. J. Org. Chem. 1999, 1999, 1253-1257; b) L. T. Eremenko, L. B. Romanova, M. E. Ivanova, I. L. Eremenko, S. E. Nefedov, Y. T. Struchkov, Russ. Chem. Bull. 1994, 43, 619-623; c) H. Irngartinger, S Strack, Acta Cryst Sect C 1994, 50, 2017-2019; d) D. S. Yufit, Y. T. Struchkov, L. T. Eremenko, Russ. Chem. Bull. 1993, 42, 1152-1155; e) P. R. Dave, R. Duddu, K. Yang, R. Damavarapu, N. Gelber, R. Surapaneni, R. Gilardi, Tetrahedron Lett. 2004, 45, 2159-2162; f) R. M. Moriarty, J. S. Khosrowshahi, R. S. Miller, J. Flippen-Andersen, R. Gilardi, J. Am. Chem. Soc. 1989, 111, 8943-8944; g) M. Hoshino, A. Khutia, H. Xing, Y Inokuma, M. Fujita, IUCrJ 2016, 3, 139-151; h) H. Irngartinger, S Strack, F. Gredel, Liebigs Ann. 1996, 1996, 311-315; i) L. T. Eremenko, L. B. Romanova, M. E. Ivanova, A. V. Shastin, I. L. Eremenko, S. E. Nefedov, Russ. Chem. Bull. 1998, 47, 441-446; j) R. M. Moriarty, M. S. C. Rao, S. M. Tuladhar, C. D'Silva, G. Williams, R. Gilardi, J. Am. Chem. Soc. 1993, 115, 1194-1196; k) Y. Inokuma, S. Yoshioka, J. Ariyoshi, T. Arai, Y. Hitora, K. Takada, S. Matsunaga, K. Rissanen, M. Fujita, Nature 2013, 495, 461-466 I) B. Harris, G. P. Savage, J. M. White, Org. Biomol. Chem. 2013 11, 3151-3158$$
1154
$$

G. R. Desiraju, J. Am. Chem. Soc. 2013, 135, 9952-9967.

G. R. Desiraju, P. S. Ho, L. Kloo, A. C. Legon, R. Marquardt, P. Metrangolo, P. Politzer, G. Resnati, K. Rissanen, Pure Appl. Chem. 2013, 85, 1711-1713.

G. R. Desiraju, R. Parthasarathy, J. Am. Chem. Soc. 1989, 111, 8725-8726.

[21] B. K. Saha, A. Nangia, J.-F. Nicoud, Cryst. Growth Des. 2006, 6, $1278-1281$.
G. Cavallo, P Metrangolo, R. Milani, T. Pilati, A Primagi, G. Resnati, G. Terraneo, Chem. Rev. 2016, 116, 2478-2601.

[23] a) H. L. Ammon, C. S. Choi, S. Reddy, Acta Cryst. Sect. C 1988 44, 1671-1672; b) N. T. Kawai, D. F. R. Gilson, J. F. Britten, I. S. Butler, P. G. Farrell, Can. J. Chem. 1992, 70, 910-914.

[24] A. V. Shastin, V. V. Zakharov, G. P. Bugaeva, L. T. Eremenko, L. B. Romanova, G. V. Lagodzinskaya, G. G. Aleksandrov, I. L. Eremenko, Russ. Chem. Bull. 2006, 55, 1304-1306.

[25] V. V. Zakharov, G. P. Bugaeva, L. S. Barinova, G. V. Lagodzinskaya, L. B. Romanova, G. G. Aleksandrov, L. T. Eremenko, I. L. Eremenko, Russ. Chem. Bull. 2005, 54, 15051508.

[26] L. T. Eremenko, L. B. Romanova, M. E. Ivanova, V. S. Malygina, L. S. Barinova, G. V. Lagodzinskaya, V. P. Lodygina, I. L. Eremenko, G. G. Aleksandrov, Russ. Chem. Bull. 2007, 56, 1408-1422.

[27] a) P. E. Eaton, K. Pramod, T. Emrick, R. Gilardi, J. Am. Chem. Soc 1999, 121, 4111-4123; b) K. Hassenruck, J. G. Radziszewski, V. Balaji, G. S. Murthy, A. J. McKinley, D. E. David, V. M. Lynch, H. D. Martin, J. Michl, J. Am. Chem. Soc. 1990, 112, 873-874.

[28] a) M. O. Senge, Chem. Commun. 2006, 243-256; b) M. O. Senge S. A. MacGowan, J. M. O'Brien, Chem. Commun. 2015, 51 17031-17063.

[29] P. E. Eaton, E. Galoppini, R. Gilardi, J. Am. Chem. Soc. 1994, 116, 7588-7596.

[30] S. D. Karlen, H. Reyes, R. E. Taylor, S. I. Khan, M. F. Hawthorne M. A. Garcia-Garibay, Proc. Natl. Acad. Sci. U.S.A. 2010, 107, 14973-14977.

[31] a) I. J. Bruno, J. C. Cole, P. R. Edgington, M. Kessler, C. F. Macrae, P. McCabe, J. Pearson, R. Taylor, Acta Crystallogr. Sect. B 2002, 58, 389-397; b) C. R. Groom, I. J. Bruno, M. P. Lightfoot S. C. Ward, Acta Cryst. Sect. B 2016, 72, 171-179.

[32] C. F. Macrae, I. J. Bruno, J. A. Chisholm, P. R. Edgington, P. McCabe, E. Pidcock, L. Rodriguez-Monge, R. Taylor, J. van de Streek, P. A. Wood, J. Appl. Crystallogr. 2008, 41, 466-470.

[33] O. V. Dolomanov, L. J. Bourhis, R. J. Gildea, J. A. K. Howard, H. Puschmann, J. Appl. Crystallogr. 2009, 42, 339-341.

[34] H. Hope, Prog. Inorg. Chem. 2007, 1-19.

[35] a) Saint, version 8.37a, Bruker AXS, Inc., Madison, WI, 2013; b) SADABS, version 2016/2, Bruker AXS, Inc, Madison, WI,, 2014; c) APEX3, version 2016.9-0., Bruker AXS, Inc., Madison, WI, 2016. G. Sheldrick, Acta Crystallogr. Sect. A 2015, 71, 3-8. 


\section{Entry for the Table of Contents}

\section{FULL PAPER}

Cubanes have been used successfully as a bioisostere for benzene rings, but most of these studies rely on the fact that the 1,4-axis of both these compounds are of similar length. However, with its 3D geometry, cubane has a significant difference in the types of interactions that occur in comparison to benzene. Herein we highlight the differences by an indepth study on cubane hydrogen interaction profiles with multiple functional groups.

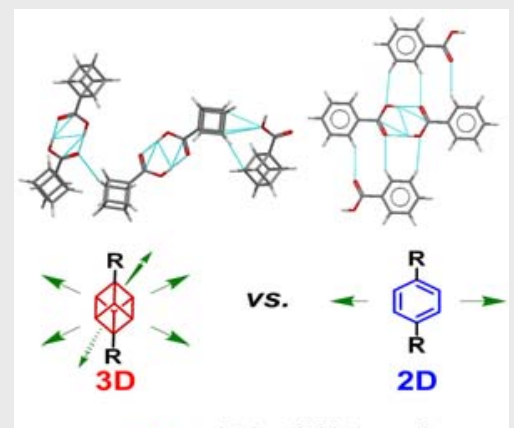

Keith J. Flanagan, Stefan S. R. Bernhard, Shane Plunkett, and Mathias O. Senge*

Page No. - Page No.

Not Your Usual Bioisostere: Solid State Study of 3D Interactions in Cubane 